\title{
Multiparametric investigation of non functionalized-AGulX nanoparticles in 3D human airway epithelium models demonstrates preferential targeting of tumor cells
}

\author{
Lucie Sancey $^{1^{*}}$ (1) Odile Sabido 2,3 , Zhiguo He ${ }^{3,4}$, Fabien Rossetti ${ }^{5}$, Alain Guignandon ${ }^{6,3}$, Valérie Bin ${ }^{2,3}$, \\ Jean-Luc Coll ${ }^{1}$, Michèle Cottier ${ }^{2,3,7}$, François Lux ${ }^{5,8,9}$, Olivier Tillement ${ }^{5,8}$, Samuel Constant ${ }^{10,11}$, Christophe Mas $^{11}$ \\ and Delphine Boudard $2,3,7^{*}$
}

\begin{abstract}
Liquid deposit mimicking surface aerosolization in the airway is a promising strategy for targeting bronchopulmonary tumors with reduced doses of nanoparticle (NPs). In mimicking and studying such delivery approaches, the use of human in vitro 3D culture models can bridge the gap between 2D cell culture and small animal investigations. Here, we exposed airway epithelia to liquid-apical gadolinium-based AGuIX ${ }^{\circledR} N P s$ in order to determine their safety profile. We used a multiparametric methodology to investigate the NP's distribution over time in both healthy and tumorbearing 3D models. AGuIX ${ }^{\circledR}$ NPs were able to target tumor cells in the absence of specific surface functionalization, without evidence of toxicity. Finally, we validated the therapeutic potential of this hybrid theranostic AGuIX ${ }^{\circledR}$ NPs upon radiation exposure in this model. In conclusion, 3D cell cultures can efficiently mimic the normal and tumorbearing airway epitheliums, providing an ethical and accessible model for the investigation of nebulized NPs.
\end{abstract}

Keywords: AGuIX ${ }^{\circledR}$ nanoparticles, 3D human healthy and tumor airway models, Nanoparticle's toxicity, Nanoparticle's uptake, Tumor targeting

\section{Background}

Non-small-cell lung cancer (NSCLC) is the principal type of lung cancer for the respiratory tract (85\%), for which the standard treatment is surgery, especially for patients with early-stage NSCLC. However, some patients are not eligible for surgery because of medical comorbidities. In such cases, radiotherapy is an option that should be considered, in particular for early-stage, node-negative

\footnotetext{
*Correspondence: lucie.sancey@univ-grenoble-alpes.fr; delphine. boudard@univ-st-etienne.fr

1 Institute for Advanced Biosciences, INSERM U1209, CNRS, UMR 5309, Université Grenoble Alpes, 38000 Grenoble, France

${ }^{2}$ INSERM U1059, Laboratoire SAINBIOSE, équipe DVH/PIB, Faculté de Médecine, Université Jean Monnet, Saint-Etienne, France

Full list of author information is available at the end of the article
}

patients [1]. The standard of care recommendations promote the stereotactic radiation (SR) approach, a modality able to precisely deliver high-dose fractions to a small target or volume of disease, thereby reducing the dose or sparing healthy tissues [2]. Among SR approaches, image-guided radiation therapy (IGRT), intensity-modulated radiation therapy (IMRT), and volumetric modulated arc therapy (VMAT) constitute recent methods aimed at improving the efficacy of radiotherapy [3].

Despite these improvements, the main constraint is limiting toxicity in healthy tissues that should be spared. Therefore, the use of radiosensitizing agents containing high- $Z$ elements such as gadolinium $(Z=64)$ [4-7], hafnium $(Z=72)[8]$, and gold $(Z=79)[9,10]$, that specifically accumulate in the tumor has great potential for

(c) The Author(s) 2020. This article is licensed under a Creative Commons Attribution 4.0 International License, which permits use, sharing, adaptation, distribution and reproduction in any medium or format, as long as you give appropriate credit to the original author(s) and the source, provide a link to the Creative Commons licence, and indicate if changes were made. The images or other third party material in this article are included in the article's Creative Commons licence, unless indicated otherwise in a credit line to the material. If material is not included in the article's Creative Commons licence and your intended use is not permitted by statutory regulation or exceeds the permitted use, you will need to obtain permission directly from the copyright holder. To view a copy of this licence, visit http://creativeco mmons.org/licenses/by/4.0/. The Creative Commons Public Domain Dedication waiver (http://creativecommons.org/publicdomain/ zero/1.0/) applies to the data made available in this article, unless otherwise stated in a credit line to the data. 
increasing the local effect of the dose deposited. From this perspective, gadolinium (Gd)-based nanoparticles (NPs) are of major interest, as they have both radiosensitizing characteristics and contrast properties for magnetic resonance imaging $[11,12]$. The challenges of using radiotherapy for the treatment of lung diseases include gating the area of interest requiring radiation exposure. Such theranostic properties, i.e., imaging ability and radiosensitization, suggest the strong potential of Gd-based NPs in the clinic [13].

To increase the lung tumor uptake of a drug, several administration routes can be used, including intravenous administration (IV) and inhalation [14, 15]. While the IV route allows vascularized tumor-specific and passive (through the EPR effect) targeting [16], it requires large volumes of drug, which increases the risk of toxicity/side effects and the treatment cost. In contrast, inhalation offers an interesting alternative that limits the administration volume, maximizes the local uptake and effect of the drug, and presents another pathway for targeting the tumor $[17,18]$. In addition, inhaled drugs may cross the lung parenchyma and reach the blood stream. Because the drug is then circulating in blood, it may return to the tumor permeable blood vessels and accumulate by the EPR effect leading to two complementary tumor targeting mechanisms $[12,19]$.

In this context, we studied the toxicity and uptake, in terms of distribution and kinetics, of a Gd-based NP named AGuIX ${ }^{\circledR}$, which has already been evaluated in phase 1b clinical trials (NCT02820454, NCT03308604) and is currently tested in phase 2 (NCT03818386 and NCT04094077) [20]. AGuIX ${ }^{\circledR}$ NPs are ultrasmall NPs $(\approx 5-\mathrm{nm})$ with MRI contrast and radiosensitizer properties and are considered theranostic nanodrugs for personalized medicine in oncology [21]. They have been tested in several preliminary in vitro and in vivo studies that evaluated their biotoxicity, biodistribution and biopersistence [4-6]. An in vivo study of localized AGuIX ${ }^{\circledR}$ intrathecal instillation in mice showed increased tumor targeting and radiotherapy efficiency compared to systemic injection [12, 18, 19]. As well, AGuIX ${ }^{\circledR}$ aerosolization may represent a promising theranostic approach for the treatment of lung tumors and metastases [22]. In order to mimic NP's liquid aerosolization, we used two innovative 3D human models of the respiratory tract, one without tumor cells (MucilAir ${ }^{\mathrm{TM}} /$ Epithelix) and one with A549 adenocarcinoma nodules (OncoCilAir ${ }^{\mathrm{TM}} /$ Oncotheis) [23].

MucilAir $^{\mathrm{TM}}$ is a fully differentiated and ready-to-use 3D model of human airway epithelium [24] made of primary human epithelial cells freshly isolated from nasal or bronchial human biopsies. MucilAir ${ }^{\mathrm{TM}}$ reproduces the morpho-functional characteristics of the original tight, polarized, pseudostratified, prismatic respiratory epithelia, with three types of cells: differentiated ciliated epithelial cells $(>50-60 \%)$, goblet cells $(10-20 \%)$ that produce a part of the mucus secretion, and basal cells (remaining fraction) that enable epithelium renewal; the basal cells may be at an undifferentiated state or undergoing processes such as ciliogenesis [25-28]. The air-liquid interface of this in vitro model accurately reproduces the physiology of human airway epithelia, including the presence of a functional mucociliary system with ciliary beating and mucus secretion in a homeostatic state [24]. This biological interface is important to consider in pharmacological and nanotoxicological experiments $[29,30]$. Similarly, OncoCilAir ${ }^{\mathrm{TM}}$ is a unique human in vitro $3 \mathrm{D}$ lung cancer model based on the coculture of human primary bronchial epithelial cells with incorporated A549GFP tumor cells in the airway tissue, which facilitates observation and tumor growth. This model reproduces as closely as possible tumor invasion of a normal environment. In our case, A549 tumor cells were selected as the lung tumor model [31].

In the present study, we performed a bio-toxicological evaluation of Gd-based AGuIX ${ }^{\circledR}$ NPs on airway MucilAir $^{\text {TM }}$ cultures, combining quantitative and qualitative NP uptake analysis through flow cytometry (FCM) approach, image acquisition (epi-fluorescence and confocal imaging), and ICP measurements [32-34]. NP exposure was achieved as restrained liquid exposure directly to the apical surface of the tissue with or without mucus interface, mimicking droplets-NP aerosolization and therefore the clinical approach. Second, we investigated and compared AGuIX ${ }^{\circledR}$ NP distribution, and internalization by tumor and healthy cells in OncoCilAir ${ }^{\mathrm{TM}}$ cultures using multiparametric investigations. Finally, the radiosensitizing effect of AGuIX ${ }^{\circledR}$ NPs was evaluated.

\section{Methods \\ AGuIX ${ }^{\circledR}$ NPs characteristics}

The Gd-based nanoparticles are made of a polysiloxane network surrounding by cyclic chelates of gadolinium [35]. Their main characteristics are their ultra-small size with a hydrodynamic diameter of $3 \pm 2 \mathrm{~nm}$ and a mass of approximately $10 \mathrm{kDa}$, a mean $\mathrm{Gd} / \mathrm{Si}$ ratio of $10 / 40$, and a strong complexation constant $\left(\log \beta_{110}\right)$ for $\mathrm{Gd}$ (i.e. 24.78). The particles were developed for medical theranostic approaches, combining the contrast properties for MRI acquisition in T1 mode, and the radiosensitizing action of the high- $Z$ element gadolinium $(Z=64)$. Different fluorescent dyes (FITC, Cy5.5, and Rhodamine-B (RhoB)) have been conjugated to the $\mathrm{AGuIX}^{\circledR}$ for the biological investigations as previously reported [36], with a fluorescent conjugation yield of $1 / 600$ to $1 / 250$. A short characterization of the NPs can be found in Additional 
file 1: Figure S1. The AGuIX ${ }^{\circledR}$ are freeze dried for longterm storage and can be solubilized in water and biocompatible solvent $(\mathrm{NaCl} 0.9 \%$, as example) before administration.

\section{Culture of MucilAir/OncoCilAir ${ }^{\mathrm{TM}}$ tissues}

MucilAir $^{\mathrm{TM}}$ and OncoCilAir ${ }^{\mathrm{TM}}$ were produced by Epithelix and OncoTheis Companies respectively (see Additional file 1: Figure S2). A complete description of the cultures can be found on the websites (Epithelix, Geneva, Switzerland, http://www.epithelix.com and Oncotheis http://www.oncotheis.com). Upon receipt, bronchial MucilAir $^{\mathrm{TM}}$ pool of donors cultures or OncoCilAir ${ }^{\mathrm{TM}} \mathrm{cul}^{-}$ tures (KRAS mutated) containing A549-GFP cells were transferred into 24-well plates filled with $700 \mu \mathrm{L}$ of specific pre-warmed MucilAir ${ }^{\mathrm{TM}}$ or OncoCilAir ${ }^{\mathrm{TM}}$ culture medium. The tissues were routinely cultured at $37^{\circ} \mathrm{C}$ and $5 \% \mathrm{CO}_{2}$ in a saturated humidity environment ( $\left.\geq 99 \%\right)$ in air-liquid interface (ALI) culture condition. Medium renewal was performed every two-three days. Transepithelial electrical resistance (TEER) was assessed as a
$1.25 \mathrm{mM} \mathrm{CaCl}_{2}(\mathrm{Vf}=30 \mu \mathrm{L})$. For apical exposure without mucus, the mucus was gently removed using a PBS washing step. All the bio-toxicity markers used for the analysis were assessed after the 24-h AGuIX ${ }^{\circledR}$ exposure.

Morphological observations of cultures before and after $\mathrm{AGuIX}^{\circledR}$ exposure were performed to evidence potentially figure modifications as decrease of ciliary frequency, detachment of cells, or apparition of cavity/ hole into the insert using an inverted optical microscope (MOTIC AE2000 Trino).

Cell mortality was then evaluated by quantifying the lactate dehydrogenase (LDH) released from cells into the basal compartment with damaged membranes using the LDH Cytotoxicity kit plus (Roche Diagnostics, Mannheim, Germany), according to the manufacturer's instructions. Optical density detection was performed using a microplate reader (Multiskan GO, Thermo Fisher Scientific Inc., Wyman Street, Waltham, USA) at $490 \mathrm{~nm}$. The amount of the released LDH was reported as a percentage of cytotoxicity according to the following equation:

$$
\text { Cytotoxicity }(\%)=\frac{(\mathrm{OD} \text { of AGuIX exposed samples }- \text { OD of unexposed samples })}{(\mathrm{OD} \text { of positive controls }- \text { OD of unexposed samples })} \times 100
$$

standard indicator of tissues integrity. Measurements were conducted using an $\mathrm{EVOM}^{\circledR}$ resistance meter and STX 2 electrodes (World Precision Instruments, Sarasota, USA) at least once a week, and before/after NPs exposure. The TEER values $(\Omega)$ were established by using the following formula: TEER $\left(\Omega \cdot \mathrm{cm}^{2}\right)=$ (resistance value $(\Omega)-100(\Omega)) \times 0.33\left(\mathrm{~cm}^{2}\right)$, where 100 is the resistance of the insert membrane and $0.33 \mathrm{~cm}^{2}$ is the total surface of the 24-well plates culture insert. The TEER values described for well-preserved MucilAir ${ }^{\mathrm{TM}}$ cultures could be $>200 \Omega \cdot \mathrm{cm}^{2}$ (well-preserved 200-600 $\Omega \cdot \mathrm{cm}^{2}$ [24] with optimal mean TEER $300-400 \Omega \cdot \mathrm{cm}^{2}$ [25]), and for OncoCilAir $^{\mathrm{TM}}$ around $100-200 \Omega \cdot \mathrm{cm}^{2}$, the presence of tumor A549 areas decreasing the tightness of the epithelium, as reported by the company.

\section{AGuIX ${ }^{\circledR}$ acute toxicity evaluation on MucilAir ${ }^{\mathrm{TM}}$}

MucilAir $^{\mathrm{TM}}$ AGuIX $^{\circledR}$ exposure has consisted of a liquid apical (air/liquid interface) or basal (liquid/liquid interface) 24-hour exposure, for final concentrations of 1 and $10 \mathrm{mM}\left(\left[\mathrm{Gd}^{3+}\right]\right) \mathrm{NPs}$ according to the experiments in their native or conjugated (FITC, RhoB, or Cy5.5) form; with kinetic monitoring up to 72-hour after apical and basal washing with PBS and addition of fresh medium in the basal compartment. AGuIX ${ }^{\circledR}$ solutions have been prepared with specific MucilAir ${ }^{\text {TM }}$ culture medium for basal exposure $(\mathrm{Vf}=700 \mu \mathrm{L})$, or for apical exposure advisable vehicle saline solution of $10 \mathrm{mM}$ Hepes, $\mathrm{NaCl} 0.9 \%$ and after subtraction for all values of blank control (OD of culture medium). The positive control corresponds to OD values after the complete lysis of control cells, with adequate buffer provided in the kit.

IL-8 pro-inflammatory response was assessed using a commercial enzyme-linked immunosorbent assay kit (Quantikine ${ }^{\circledR}$ Human IL-8 Immunoassay, R\&D systems Inc., McKinley Place NE, Minneapolis, USA). The optical density was determined according to the manufacturer's instructions, using a microplate reader (Multiskan GO, Thermo Scientific) at $450 \mathrm{~nm}$. A standard curve was established, and results were expressed as $\mathrm{pg} \mathrm{mL}^{-1}$ of IL-8. A specific IL-8 positive control (cytomix stimulation: TNF-alpha at $500 \mathrm{ng} / \mathrm{mL}$ and LPS at $200 \mu \mathrm{g} / \mathrm{mL}$ incubated for $24-\mathrm{h}$ in the basal culture medium) was used, according to the manufacturer recommendations [37]. It allowed the production of $4400 \pm 510 \mathrm{pg} / \mathrm{mL}$ IL-8 $(p=0.028$ compared to unexposed cells). Possible interaction of the NPs and the assays was checked to ensure the absence of false negative results.

\section{AGuIX $^{\circledR}$ permeability assessment on MucilAir ${ }^{\mathrm{TM}}$ barrier}

To assess the apparent permeability $\left(\mathrm{P}_{\text {app }}\right)$ of a compound across upper-airway epithelium of a respiratory tract, we specifically investigate the apical-to-basal transport of AGuIX-FITC in MucilAir ${ }^{\mathrm{TM}}$ tissues, using the same NP's preparation as described in "AGuIX ${ }^{\circledR}$ acute toxicity 
evaluation on MucilAir ${ }^{\mathrm{TM}}$ " section. For this experiment, only inserts displaying a TEER around $300 \Omega \cdot \mathrm{cm}^{2}$ were used. Atenolol $(10 \mathrm{mM})$ and salicylic acid $(100 \mu \mathrm{M})$ were respectively used as low and high chemical permeability molecules, and the values were compared with the one of AGuIX $^{\circledR}$ exposure [24, 38]. All compounds were diluted in HBSS transport buffer. Prior to incubation, all solutions were pre-warmed to $37^{\circ} \mathrm{C}$ and the $\mathrm{pH}$ was adjusted to 7.4. Each condition was evaluated in triplicate. $\mathrm{P}_{a p p}$ was determined as follow. A pre-warmed HBSS donor solution $(200 \mu \mathrm{L})$ was added to the apical compartment. A fraction of the donor solution was immediately withdrawn to determinate the effective initial concentration or fluorescence (AGuIX ${ }^{\circledR}$-FITC). At the same time, 500 $\mu \mathrm{L}$ HBSS solution were filled into the basal acceptor compartment. Transport experiments were performed during $2 \mathrm{~h}$ at $37^{\circ} \mathrm{C}$. The fluorescent intensity of AGuIX ${ }^{\circledR}$-FITC into apical and basal AGuIX ${ }^{\circledR}$-FITC after this permeability experiment were measured with a fluorometer (Fluoskan Ascent, Thermo Fisher Scientific Inc., Wyman Street, Waltham, USA). The amount of atenolol and salicylic acid that permeated through the culture insert in both compartments was quantified by liquid chromatography-mass spectrometry (Aquity UPLC system) coupled with a Xevo TQ-D triple quadrupole mass spectrometer (Waters, Saint-Quentin-en-Yvelines, France). The permeability was finally calculated using the following equation:

$$
\text { Papp }=\frac{V}{C i \times A} \times \frac{C f}{\Delta t}
$$

where $\mathrm{V}$ is the volume of the donor compartment $\left(\mathrm{cm}^{3}\right)$, $\mathrm{C}_{\mathrm{i}}$ is the initial concentration of the compound (in $\mathrm{g} / \mathrm{L}$ or $\mathrm{M})$ or fluorescence intensity, $\mathrm{A}$ is the area of the insert $\left(0.33 \mathrm{~cm}^{2}\right), \mathrm{C}_{\mathrm{f}}$ is the final concentration of the compound in the acceptor compartment (in $\mathrm{g} / \mathrm{L}$ or $\mathrm{M}$ ) or fluorescence intensity, and $\Delta \mathrm{t}$ is the duration of the experiment (in s). Papp was expressed as $\mathrm{cm} \cdot \mathrm{s}^{-1}$.

\section{Functional monitoring of MucilAir ${ }^{\mathrm{TM}}$ cultures}

Measurement of the Cilia Beating Frequency (CBF) was performed at room temperature (RT) by a dedicated setup made of three parts: a camera (Sony XCD V60 Firewire), a PCI card, and a specific package of software. The CBF was calculated using CiliaX software (Epithelix, Geneva, Switzerland), and expressed as $\mathrm{Hz}$.

The mucociliary clearance was monitored using a high-speed acquisition camera (Sony) connected to an Axiovert $200 \mathrm{M}$ microscope (Zeiss, Jena, Germany). Microbeads (30 $\mu \mathrm{m}$ of diameter) were added onto the apical surface of the MucilAir ${ }^{\mathrm{TM}}$. Then, $30 \mathrm{~s}^{\prime}$ movies (3 movies/insert) showing the movement of the small beads will be taken and analyzed using the imaging software Image Pro Plus (Mediacy). The movement of the beads was tracked, and velocity of each particle was calculated to determine the speed of the mucociliary clearance.

\section{MucilAir $^{\mathrm{TM}}$ phenotypic analysis by Flow Cytometry (FCM)}

According to Epithelix company, MucilAir ${ }^{\mathrm{TM}}$ tissues were dissociated to surface insert with a trypsinization protocol to obtain a cellular suspension containing approximately 300000 cells/insert. For each immunophenotyping, 100,000 cells were used. To avoid fixation/ permeabilization steps inducing a significant loss of the intracellular AGuIX ${ }^{\circledR}$ fluorescent signal, the cell type discrimination was performed with a vital CLCA1 staining (anti-CLCA1.PE-Cy5.5 human polyclonal goat antibody, \#AC21-1575-16, Abcore, CA). CLCA1 is a membrane marker expressed in mediating calcium-activated chloride conductance for mucus production by goblet cells, whose specificity could be assessed in a previous MucilAir $^{\text {TM }}$ characterization study $[25,26]$. This immunolabeling allowed a discriminant gating between the two differentiated cell types of CLCA $1^{+}$goblet population $v s$. CLCA $1^{-}$population, that included mostly ciliated cells and at last basal cells. Finally, for each sample, 50,000 cells were analyzed by flow cytometry (FCM), and the fluorescent signal was considered as quantitative. Intact cells were selected by a sequential gating using the FSC vs. SSC cytogram before selection according to specific $\mathrm{CLCA}^{+}{ }^{-}$vs. CLCA1 ${ }^{-}$cell populations. Gain setting was established by running unstained and isotype controls cells (Lightning-link PE-Cy5.5 tandem conjugation kit to IgG isotype control antibody, Innova Biosciences). AGuIX $^{\circledR}$-FITC or GFP, green fluorescence, excited by $488 \mathrm{~nm}$ laser line was acquired through a BP $530 / 30 \mathrm{~nm}$ filter, PE-Cy5.5 excited by $488 \mathrm{~nm}$ laser line, through a $695 / 40 \mathrm{~nm}$ BP filter, and far red fluorescence of Cy5.5, excited by $633 \mathrm{~nm}$ laser line, through a 712/21 nm BP filter. Compensations were calculated by acquisition of fluorescent beads of each used fluorophore (Calibrat ${ }^{\mathrm{TM}}$ $\mathrm{BD}$ Biosciences, CA). Both percentage and geometric mean of fluorescence were collected. The results were expressed as the percentage of cells of interest, and geometric mean fluorescence intensity (MFI). FACS DiVa (BD Biosciences, CA) equipped with an argon ion and $\mathrm{He}-\mathrm{Ne}$ lasers (Coherent, $\mathrm{CA}$ ) was used. Data were analyzed with DiVa 5.03 software.

\section{Quantitative NPs' uptake evaluation by FCM}

Tumor cell population on OncocilAir ${ }^{\mathrm{TM}}$ model was ranked by FCM according to the GFP fluorescent signal intensity. The total cell population was therefore split into 3 sub-populations, i.e., normal healthy cells $\left(\mathrm{GFP}^{-}\right), \mathrm{GFP}^{+}$ and $\mathrm{GFP}^{++}$tumor cells, expressing respectively low and high level of GFP protein. For each sub-population, the level of $\mathrm{AGuIX}^{\circledR}$ uptake was determined and expressed as 
the $\%$ of positive cells and MFI values. Finally, a relative uptake ratio values between tumor and normal areas was calculated as follow, after deduction of autofluorescence values:

$$
A G u I X \text { relative uptake ratio }=\frac{\left(M F I_{G F P+,++}\right)}{\left(M F I_{G F P-}\right)}
$$

\section{Cell cycle analyzed by FCM}

The cell cycle profile was determined according the double staining of DNA A-T specific vital dye Hoechst combined with propidium iodide (PI), a passive DNA intercalating dye. Hoechst fluorescence was collected after a UV excitation, while emission was collected through a $424 / 44 \mathrm{~nm}$ BP filter, and PI was excited at $488 \mathrm{~nm}$, and its emission collected through a 695/40 nm BP filter. Dead cells and doublets of cells were excluded based upon PI positive staining, and specific gating drawing based upon Hoechst width signals versus Hoechst area signals respectively. Quiescent $\left(\mathrm{G}_{0} / \mathrm{G}_{1}\right)$ and proliferative ( $S$ and $G_{2}+M$ ) cells were determined on the basis of linear quantification of Hoechst fluorescence.

\section{Microscopic observations of flat mounted MucilAir ${ }^{\mathrm{TM}}$ / OncoCilAir $^{\mathrm{TM}}$ tissues \\ Tissue preparations and observations}

For observations of living tissues, the whole living MucilAir $^{\mathrm{TM}} /$ OncoCilAir ${ }^{\mathrm{TM}}$ tissues was removed from its cell culture insert and placed on a microscopic slide. A Viscoelastic System (Viscot, DuoVisc, Alcon) containing hyaluronate de sodium, chondroitin sulfate was added on the tissue for minimizing the cell stress during coverslip mounting. The tissue was then gently flattened using a large glass coverslip retained by adhesive tape.

\section{Epifluorescence microscopy}

By using an epifluorescence inverted microscope IX81 (Olympus, Tokyo, Japan) equipped with the CellSens imaging software (Olympus, Munster, Germany), the cells were visualized by phase-contrast, and tumor cells were highlighted by the GFP-fluorescence.

\section{Confocal microscopy}

The internalization of AGuIX ${ }^{\circledR}$-Cy5.5 was relatively evaluated for A549-GFP tumor cells and healthy surrounding epi-airway epithelium using a FLUOVIEW FV1200 laser scanning confocal microscope (Olympus, Tokyo, Japan) equipped with the FV10-ASW4.1 imaging software. A $635 \mathrm{~nm}$ laser was used to excite the Cy5.5 dye, while the signal was collected using a long-pass filter $\lambda>650 \mathrm{~nm}$.

\section{Tumor-nodule area monitoring on OncoCilAir ${ }^{\mathrm{TM}}$}

The growth of A549 tumor nodules tagged by GFP was observed on whole surface of OncoCilAir ${ }^{\mathrm{TM}}$ without any sample preparation, using a fluorescence macroscope MVX10 (Olympus, Tokyo, Japan) equipped with the software CellSens imaging systems (Olympus, Hamburg, Germany) or the macroscope system Leica equipped with the software Andor SOLIS (Oxford Instruments).

\section{Measurement of tumor area surface on OncoCilAir ${ }^{\mathrm{TM}}$}

From images acquired with Olympus fluorescence macroscope at the time culture reception (i.e. 20-25 days after A549 cell implantation) and during their followup, an image processing using ImageJ platform has been performed (open-source program of the National Institutes of Health, http://rsb.info.nih.gov/ij/). Eight [8] bits raw images were first treated to remove the background and tumor edges were detected (edge filtering). Raw and edges images were combined to increase automatic detection of tumor areas. Detection of tumor areas was performed by applying a Reny threshold. Binary areas were sent to ROI manager to extract individually settings: area, perimeter, mean fluorescent intensity of all detected tumors. The mean fluorescent intensity signal of 100 over 255 was chosen to discriminate $\mathrm{GFP}^{+}$and $\mathrm{GFP}^{++}$populations. Mean signals under 100 were identified as $\mathrm{GFP}^{+}$, and $\mathrm{GFP}^{++}$was attributed for values $>100$. Individual temporal tracking of tumors areas and intensities were exported to GraphPad Prism7 for figures and statistical analysis.

\section{Additional nanoparticle observations by two-photon confocal microscopy}

AGuIX $^{\circledR}$-RhoB (10 mM) were added to the apical phase of the cells during the indicated time. Two-photon microscopy was performed as described in Sancey et al. [39], using a LSM 7 MP (Zeiss, Germany) equipped with a $20 \times$ water-immersion objective (NA 1.0; Zeiss) and ZEN 2010 software for detection of the NPs. Laser excitation was done at $800 \mathrm{~nm}$ with a Ti:Sapphire laser (Chameleon vision II; Coherent, UK). Fluorescence emissions were detected simultaneously by three non-descanned photomultiplier tubes with a 492/SP25 nm filter (Semrock, US) for blue autofluorescence and Hoechst emission, a $542 / 50 \mathrm{~nm}$ filter (Semrock, US) for green autofluorescence emission, and a $617 / 73 \mathrm{~nm}$ filter (Semrock, US) for AGuIX $^{\circledR}$-RhoB fluorescence emission. Autofluorescence and second harmonic generation of biological structures could also be collected in the 3 channels due to the presence of collagen, lectin and elastin as example. Confocal microscopy was performed using an LSM 510 (Zeiss Germany) equipped with a $40 \times$ oil-immersion objective (NA 1.2; Zeiss). Laser excitations/emissions were $760 \mathrm{~nm}$ 
biphotonic/400-450 $\mathrm{nm}$ for Hoechst, $488 \mathrm{~nm} / 500$ $550 \mathrm{~nm}$ for FITC/GFP, $543 \mathrm{~nm} / 550-600 \mathrm{~nm}$ for Rhodamine-B, $633 \mathrm{~nm} / 650-705 \mathrm{~nm}$ for Cy-5, respectively.

\section{Immuno-labeling for confocal microcopy}

To discriminate the cellular organization of tumor areas from healthy tissue in OncoCilAir ${ }^{\mathrm{TM}}$ model, the sample was fixed in 4\% paraformaldehyde (PFA) for $30 \mathrm{~min}$ at RT, then permealized in $0.5 \%$ Triton X-100 for 15 min at RT. The cells were stained with either ethidium bromide (FT25810A, Molecular Probes) or phalloidin (Actin-stain ${ }^{\text {TM }}$ 555, PHDH1, Cytoskeleton) for $30 \mathrm{~min}$ at RT, for nuclear and F-actin staining respectively. Phalloidin stained the cell outline of the respiratory epithelium. Before microscopic observations, the tissues were placed on a glass slide, covered with an anti-fading mounting medium (H1000, VECTASHIELD ${ }^{\circledR}$, Vector) and gently flattened using a large glass coverslip retained by adhesive tape.

The inserts were incubated with AGuIX ${ }^{\circledR}$ solution at $10 \mathrm{mM}$ for $60 \mathrm{~min}$ to $72 \mathrm{~h}$, in air/ $/ \mathrm{CO}_{2} 5 \%, 37^{\circ} \mathrm{C}$ environment. The inserts were cryofixed using liquid nitrogen vapor, and sliced at $8 \mu \mathrm{m}$. A solution of $5 \%$ goat serum in PBS was used to block any unspecific sites. To discriminate ciliated and goblet cells, a beta IV tubulin antibody was used (\#T6793, 1/500, Sigma-Aldrich, France) and anti-mouse IgG-Alexa488 (\#A11029, 1/2000, Life Technology, France). Beta IV tubulin is a major constituent of microtubules in motile ciliary axonemes. The sections were incubated with $1 \mathrm{mM}$ Hoechst solution before mounting with the coverslip.

\section{Quantification of Gd content by inductively coupled plasma-optical emission spectrometry (ICP-OES)}

Determination of the Gd content in the samples (apical and basal fractions, collected first after 24 h-exposure and secondly at $72 \mathrm{~h}$ end kinetic) was performed by ICPOES analyses (Varian 710-ES) with a detection limit of $0.5 \mu \mathrm{g} / \mathrm{L}$. The apical fraction was collected by gentle washing using saline solution, while basal fraction was sampling during medium renewal. Before measuring Gd concentration, $3 \mathrm{~mL}$ of aqua regia (mixture of acids: nitric and hydrochloric) were added to each fraction before heating for $3 \mathrm{~h}$ at $80{ }^{\circ} \mathrm{C}$ (SCP Science DigiPREP MS). After complete mineralization, the samples were diluted with $\mathrm{HNO}_{3}(5 \%$, w/w) to reach a 50 or $10 \mathrm{~mL}$-volume (respectively), and finally filtered at $0.2 \mu \mathrm{m}$ for the measurements (i.e. a dilution factor of 500). The results were expressed as the total mass of $\mathrm{Gd}$ in the sample or its percentage relative to the administrated dose.

\section{Cultures radiation exposure and $\mathrm{\gamma H} 2 \mathrm{AX}$ labeling}

The cultures were incubated or not with the AGuIX ${ }^{\circledR}$ NPs (10 mM apical) $24 \mathrm{~h}$ before the radiation exposure, in presence of mucus. The $\mathrm{x}$-ray exposure was performed at $4 \mathrm{~Gy}$ at $250 \mathrm{kVp}$; this dose was selected based on previous experiments. Similar experiments were performed on A549 2D cultures and the radiation exposure was conducted at 4 and $6 \mathrm{~Gy} . \gamma \mathrm{H} 2 \mathrm{AX}$ labeling was performed on fixed cells as described in Kotb et al. [4], using anti-phospho-histone H2AX (Merck Millipore). Colony assay was also performed as described in Kotb et al. [4].

\section{Statistics}

The Mann-Whitney test was used for the statistical analysis using Prism software for bio-toxicity and uptake experiments. Temporal tracking of tumors areas was compared using an ordinary one-way ANOVA with a Tukey test corrected for multiple comparisons as post hoc tests using a Graph Pad Prism7 suite.

\section{Results}

Biotoxicity evaluation of $\mathrm{AGuIX}^{\circledR} \mathrm{NPs}$ on MucilAir ${ }^{\mathrm{TM}}$

The synthesis of the gadolinium-based NPs AGuIX ${ }^{\circledR}$ has been described previously; the main characteristics are available in Additional file 1: Figure S1 [21]. The administration of $\mathrm{AGuIX}^{\circledR}$ NPs through the airways has been reported in vivo in mice with the hypothesis of both direct uptake and indirect vascular uptake after NP passage into the epithelium and blood stream [12]. Before investigating this phenomenon in details, the toxicity of AGuIX ${ }^{\circledR}$ NPs was evaluated based on different and complementary biological parameters in healthy airway MucilAir ${ }^{\mathrm{TM}}$ inserts, according to previous nanotoxicological studies $[40,41]$. The results summarized in Table 1 revealed that AGuIX ${ }^{\circledR}$ NPs did not cause any loss of integrity of MucilAir ${ }^{\mathrm{TM}}$ epithelium or acute biological toxicity (see Table 1), even at the high dose of $10 \mathrm{mM}$, after a 24-hour direct exposure. However, a significant decrease in TEER values was observed for AGuIX ${ }^{\circledR} \mathrm{NP}$ after apical exposure $(p<0.05)$, regardless of the AGuIX ${ }^{\circledR}$ NP dose or presence/absence of mucus. Yet TEER values had a similar profile in the saline vehicle negative control (NC) condition without mucus, indicating a stressful condition. This effect was certainly due to the condition of apical incubation with the saline solution, which acted as a cellular stress factor observed also in the NC condition especially without mucus. However, the integrity of the epithelium was not affected, even at the higher $(10 \mathrm{mM}) \mathrm{AGuIX}^{\circledR}$ NPs concentration, as the TEER values remained constant and clearly superior to the $200 \Omega \cdot \mathrm{cm}^{2}$ lower threshold value. More interestingly, the $\mathrm{AGuIX}^{\circledR}$ NPs did not have any impact on the functionality of the mucociliary system, based on mucociliary clearance and cilia beating frequency (CBF) analyses. Finally, the permeability (Papp) with or without AGuIX ${ }^{\circledR}$ NPs was comparable and $>10^{-7} \mathrm{~cm} \cdot \mathrm{s}^{-1}$, resulting in a stable Papp value 


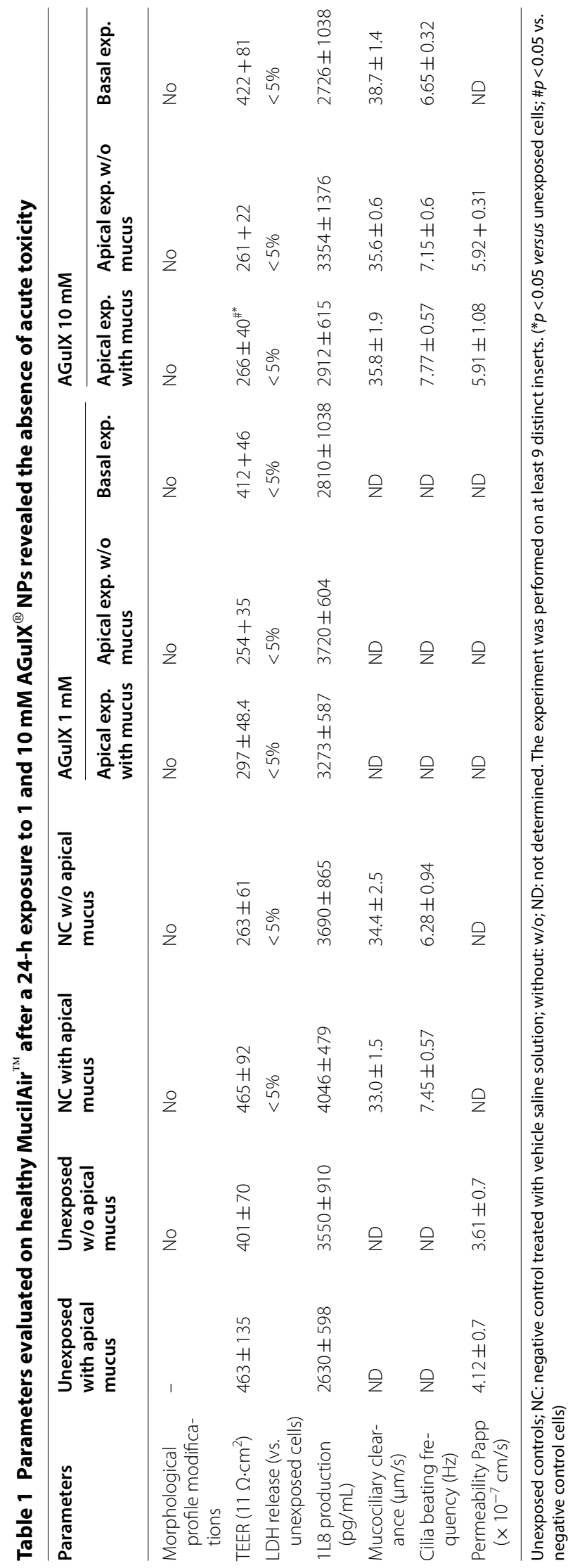


which appears even weaker than both control permeability molecules (high permeability molecule salicylic acid, Papp $=1.91 \times 10^{-5} \mathrm{~cm} \cdot \mathrm{s}^{-1}$; low permeability molecule atenolol, Papp $=2.64 \times 10^{-6} \mathrm{~cm} \cdot \mathrm{s}^{-1}$ ). So, an extremely weak MucilAir ${ }^{\mathrm{TM}}$ permeation was observed in presence of AGuIX ${ }^{\circledR}$ NPs $\approx$ fivefold lower as compared to the one of atenolol which is an hydrophilic compound characterized by a permeability among the lowest established, involving passive paracellular way for transport across MucilAir ${ }^{\mathrm{TM}}$ barrier [38]. Due to the ultrasmall size of $\mathrm{AGuIX}^{\circledR}$ NPs and previous investigations [19] these results suggest a transcellular AGuIX ${ }^{\circledR}$ passage through the epithelium. The overall results of this morpho-functional evaluation demonstrated the absence of toxicity and the safety of AGuIX ${ }^{\circledR}$ NPs under the tested conditions. Moreover, the presence or absence of mucus under apical exposure conditions had no impact on the toxicity of ultrasmall AGuIX $^{\circledR}$ NPs.

\section{Monitoring AGuIX ${ }^{\circledR} \mathrm{NP}$ behavior in healthy airway epithelium by FCM}

To mimic the direct administration of NPs by aerosolization, AGuIX ${ }^{\circledR}$ NP exposure was restricted to liquid apical distribution in the presence of mucus (physiological condition) at the higher concentration of $10 \mathrm{mM}$ in the next experiments (Fig. 1). The distribution of FITC-conjugated AGuIX $^{\circledR}$ NP was evaluated in the total viable population by FCM after 24 and $72 \mathrm{~h}$ of exposure ( $\mathrm{n}=3$ independent experiments). FCM analysis showed that approximately $15 \pm 2 \%$ of the MucilAir ${ }^{\mathrm{TM}}$ cell suspension exhibited a FITC fluorescence signal after a 24-h exposure (Fig. 1a, b, $\mathrm{d}, \mathrm{f})$. FITC fluorescence decreased heterogeneously with time, reaching almost $9 \pm 6 \%$ at $72 \mathrm{~h}$ (Fig. 1c, e, f).

To identify the cell populations involved in AGuIX ${ }^{\circledR}$ NP uptake, live cells were specifically labeled to discriminate ciliated cells from goblet cells. Of note, fixation and permeabilization were excluded to avoid major NP loss
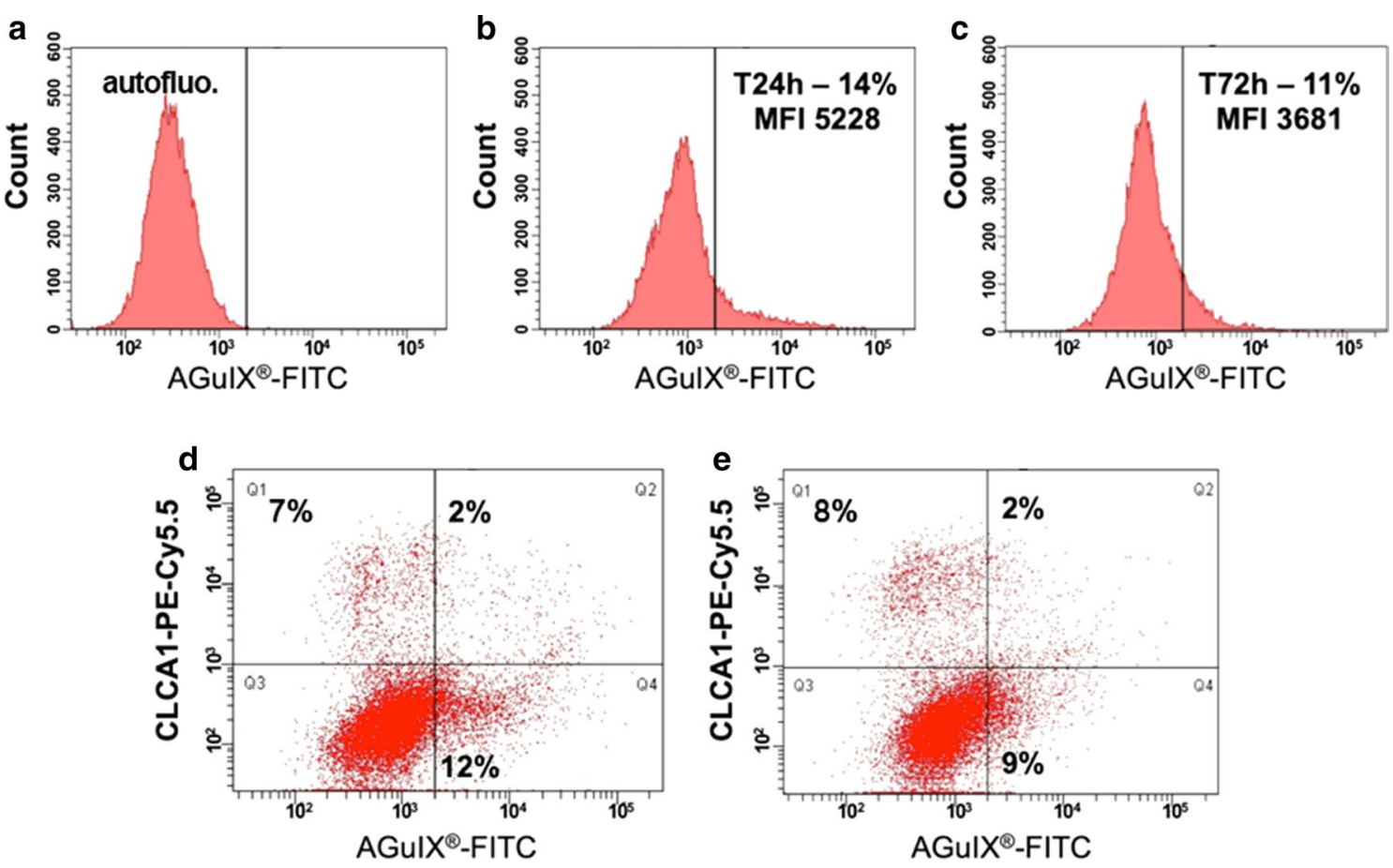

\begin{tabular}{|c|c|c|c|}
\hline Time (h) & Total $\%$ cell & $\% \mathrm{CLCA}^{+}$ & $\% \mathrm{CLCAl}^{-}$ \\
\hline 24 & $15 \pm 2$ & $2 \pm 1$ & $10 \pm 3$ \\
\hline 72 & $9 \pm 6$ & $1.5 \pm 0.5$ & $6 \pm 3$ \\
\hline
\end{tabular}

Fig. 1 FCM quantification of AGuIX ${ }^{\circledR}$ uptake in MucilAir ${ }^{\mathrm{TM}}$ cultures indicated a weak uptake in ciliated and basal cells. Representative histograms from one of the 3 experiments involving (a) control cells and AGuIX ${ }^{\circledR}$-FITC-exposed cells for (b) 24 and (c) $72 \mathrm{~h}$. The percentage of FITC-positive cells is presented as the geometric mean fluorescence intensity (MFI). Representative dot-plots of CLCA1 versus AGuIX ${ }^{\circledR}$ signal at (d) 24 and (e) $72 \mathrm{~h}$, with respectively 9\% CLCA1-positive cells including 2\% AGulX ${ }^{\circledR}$-positive versus 12\% CLCA1-negative/AGulX-positive cells and 10\% CLCA1-positive cells including $2 \%$ AGuIX ${ }^{\circledR}$-positive versus 9\% CLCA1-negative/AGulX-positive cells, and (f) the corresponding number of AGuIX ${ }^{\circledR}$-positive cells expressed as $\% \pm S D$ (mean of 3 experiments) 
during the washing steps (>50-80\%, data not shown). The mucus-producing goblet cells were identified using an anti-CLCA1 antibody [25]. According to this characterization, $11 \pm 2 \%$ of the live cells were $\mathrm{CLCA}^{+}$and considered goblet cells (Fig. 1d-e).

As described in Fig. 1f, during an additional $48 \mathrm{~h}$ of culture after the NP exposure period, the FITC signal of the NPs decreased from $15 \pm 2 \%$ at 24 h to $9 \pm 6 \%$ at $72 \mathrm{~h}$. This reduction in FITC signal was mainly observed in the $\mathrm{CLCA}^{-}$cell population, which showed a decrease from $10 \pm 3 \%$ to $6 \pm 3 \%$, while almost $2 \%$ of $\mathrm{CLCA}^{+}$cells presented a constant level of fluorescence. As indicated by the FCM analysis, AGuIX ${ }^{\circledR}$ NPs were weakly internalized by healthy cells in the MucilAir ${ }^{\mathrm{TM}}$ airway epithelium and preferentially accumulated in $\mathrm{CLCA}^{-}$cell contingent (main ciliated population and basal cells) compared to CLCA1 ${ }^{+}$goblet cells.

\section{Confirmation of AGuIX ${ }^{\circledR}$ NP distribution in the MucilAir ${ }^{\mathrm{TM}}$ model by confocal microscopy}

Native MucilAir $^{\mathrm{TM}}$ inserts were incubated with AGuIX $^{\circledR}$-RhoB and observed by intravital 2-photon microscopy (also see Additional file 1: Figure S3 for control condition). After a 1-hour incubation, the NPs were mostly observed in small cellular vesicles (Fig. 2a), away from the cilia, which was evidenced by the second harmonic generation of their components (collagen as an example). Labeling with a tubulin marker enabled the specific discrimination of the ciliary apparatus of the epithelium and the characterization of differentiated cilia cells. As illustrated in Fig. 2b, d, $1 \mathrm{~h}$ after apical exposure, the NPs accumulated in the apical pole of ciliated cells close to the cilia base and inside small cytoplasmic vesicles. Nonetheless, a strong RhoB signal was observed in the insert membrane itself, as indicated by the phase

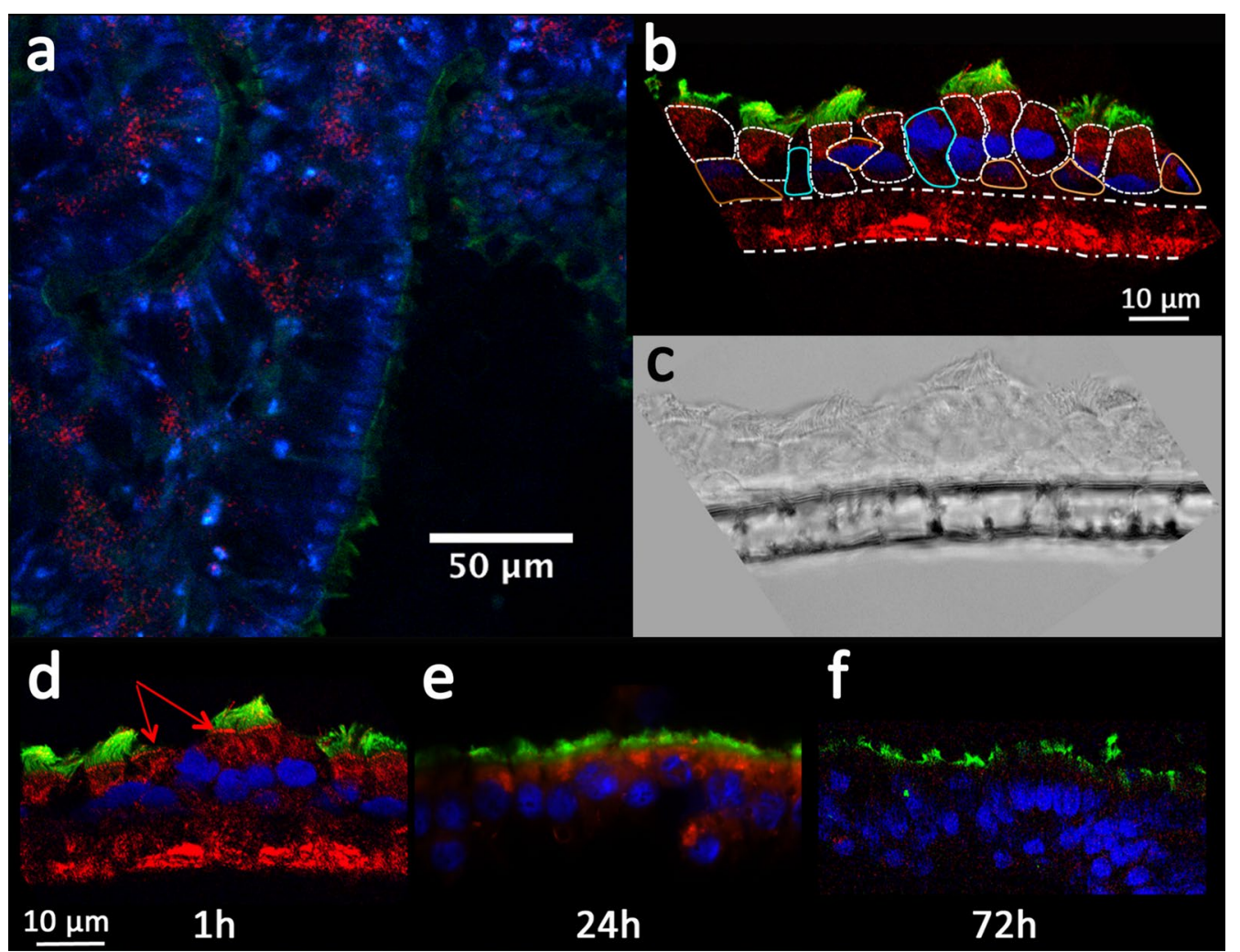

Fig. 2 Overview of AGuIX ${ }^{\circledR}$ NPs uptake and distribution on MucilAir ${ }^{\mathrm{TM}}$ by confocal microscopy evidenced the uptake in ciliated cells. a The AGul $X^{\circledR}$-RhoB solution was added at $10 \mathrm{mM}$ for $1 \mathrm{~h}$ and removed for observation using 2-photon confocal microscopy. Autofluorescence and second harmonic generation revealed structures such as cilia of ciliated cells (pseudocolored in green). Blue nuclear staining evidenced the nucleus. AGuI $X^{\circledR}$-RhoB NPs were heterogeneously distributed, as indicated by the red spots of cell vesicles. $\mathbf{b}$ Confocal layout showing the distribution of AGulX ${ }^{\circledR}$-RhoB NPs $1 \mathrm{~h}$ after apical exposure, with red arrows indicating AGuIX ${ }^{\circledR}$ NP accumulation at the base of the cilia apparatus and $\mathbf{c}$ phase contrast of a MucilAir ${ }^{\mathrm{TM}}$ insert. The ciliated cells were identified by tubulin staining (green), the nuclei are pseudocolored in blue, and the NPs are represented in red. The ciliated cells are delineated by a dotted line (white), while the goblet cells are delineated with a blue continuous line, and the basal cells are delineated with an orange continuous line. The insert membrane is indicated by an irregular dotted line. $\mathbf{d}$-f Confocal images of AGuIX ${ }^{\circledR}$ NP distribution with time: $1 \mathrm{~h}, 24 \mathrm{~h}$, and $72 \mathrm{~h}$. The red signal of the NPs decreased during the $24-72 \mathrm{~h}$ kinetic period until it was almost completely eliminated at $72 \mathrm{~h}$ after exposure 
contrast layout (Fig. 2b-c). Therefore, at early incubation times (1 to $24 \mathrm{~h}$ ), AGuIX ${ }^{\circledR}$ NPs were mostly entrapped in the plastic membrane of the insert, between the apical and basal compartments, as well as in ciliated cells. After $24 \mathrm{~h}$-exposure, goblet and basal cells were poorly positive (Fig. 2b), with preferential internalization into ciliated cells, confirming the cytometry data (CLCA1-negative cells). The microscopy and Papp data were consistent with previous studies $[19,38]$ related to transient transcytosis mechanism; this mechanism allows $\mathrm{AGuIX}^{\circledR} \mathrm{NPs}$ to be carried from the apical compartment to the basal compartment, as evidenced by the progressive decrease in the RhoB signal (Fig. 2d-f). After $72 \mathrm{~h}$, the fluorescence signal of $\mathrm{AGuIX}{ }^{\circledR}$-RhoB was almost undetectable by confocal microscopy.

\section{Quantification of AGuIX ${ }^{\circledR}$ NP contents using ICP}

Gd content was quantified by ICP measurements in the basal and apical fractions. As summarized in Table 2, the NPs were detected in both the apical and basal compartments after apical exposure, regardless of the presence of mucus. In particular, at $24 \mathrm{~h}$, almost $60 \%$ of the initial AGuIX ${ }^{\circledR}$ NP content remained in the apical fraction, while $14 \%$ was in the basal fraction, illustrating a reduced transcellular passage of NPs through the airway epithelium. In accordance with the microscopy observations on MucilAir ${ }^{\mathrm{TM}}$, very weak release of NP content was still observed at $72 \mathrm{~h}$ in the apical fraction of the same samples $(<0.5 \%)$, and the main content was measured in the basal fraction $(\approx 6 \%)$. The remaining NPs were probably entrapped in the cells and in the plastic membrane of the inserts before passing into the basal compartment (see "Confirmation of AGuIX ${ }^{\circledR}$ NP distribution in the MucilAir ${ }^{\mathrm{TM}}$ model by confocal microscopy" section). However, the limited number of cells $(\approx 300,000)$ prevented us from achieving a reliable quantification of $\mathrm{Gd}$ from the cell pellets.

\section{Characterization of the OncoCilAir ${ }^{\mathrm{TM}}$ airway model}

OncoCilAir $^{\mathrm{TM}}$ cultures are similar to the MucilAir ${ }^{\mathrm{TM}}$ model but are enriched with GFP-expressing A549 human lung tumor cells cocultured with primary bronchial fibroblasts. The healthy OncoCilAir ${ }^{\mathrm{TM}}$ surrounding tissue is preserved, without alteration of the phenotype compared to MucilAir ${ }^{\mathrm{TM}}$ cultures, although the average TEER value was significantly lower than that in MucilAir ${ }^{\mathrm{TM}}$ cultures $\left(156 \pm 34 \Omega \cdot \mathrm{cm}^{2}\right.$ vs. $463 \pm 135 \Omega \cdot \mathrm{cm}^{2}, \mathrm{n}=16 /$ condition, $p<0.001)$ and was associated with a weaker ciliary beat frequency.

The cultures were observed by fluorescence microscopy over time to determine their characteristics and evolution. In these cultures, tumor cell aggregates organized as spheroids or more or less compact clusters, as illustrated by optical and macroscopic observations (Fig. 3ab). The GFP signal allowed the discrimination of healthy cells (GFP-negative cells) and the identification of two different tumor subpopulations expressing low $\left(\mathrm{GFP}^{+}\right)$ or high levels $\left(\mathrm{GFP}^{++}\right)$of GFP. The percentage of each GFP subpopulation varied with time as the tumors grew (Fig. 3c-h). A representative tumor evolution is described in Fig. 3d, which shows the tumor area recorded at day 0 (culture reception) and at one and two weeks later. The $\mathrm{GFP}^{++}$cell population had a significantly faster proliferation rate than the $\mathrm{GFP}^{+}$cell population, as indicated in Fig. 3d on 3 separate series, independently of the percentage of the tumor area in the culture.

FCM analysis performed for the 24-72 h AGuIX ${ }^{\circledR}$ exposure indicated that GFP-negative cells accounted for $79 \pm 9 \%$ of all cells at $24 \mathrm{~h}$ and $74 \pm 12 \%$ at $72 \mathrm{~h}$; the corresponding percentages were $5 \pm 2 \%$ and $6 \pm 4 \%$ for $\mathrm{GFP}^{+}$ cells and $11 \pm 5 \%$ and $13 \pm 8 \%$ for the $\mathrm{GFP}^{++}$subpopulation (Fig. 3c-e, $n=6 /$ condition, in triplicate).

To better describe the cell subpopulations with different GFP expression (Fig. 3e, $n=3$ ), we characterized by FCM their proliferative capacity by studying the cell cycle (Fig. $3 \mathrm{f}-\mathrm{h})$. Cells in the proliferative phase made up a very weak percentage of the GFP-negative population $(2.5 \pm 0.5 \%)$ compared to the $\mathrm{GFP}^{+}(19 \pm 2 \%)$ and $\mathrm{GFP}^{++}$

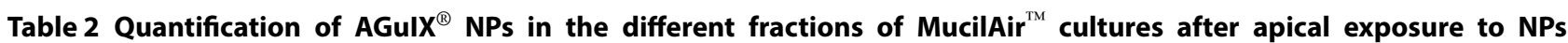
at $10 \mathrm{mM}$ demonstrated the fast clearance of healthy tissue

\begin{tabular}{|c|c|c|c|c|}
\hline \multirow[t]{2}{*}{ Conditions } & \multicolumn{2}{|l|}{$24 \mathrm{~h}$} & \multicolumn{2}{|l|}{$72 \mathrm{~h}$} \\
\hline & Apical fraction (\%) & Basal fraction (\%) & Apical fraction (\%) & $\begin{array}{l}\text { Basal } \\
\text { fraction } \\
(\%)\end{array}$ \\
\hline With mucus & $61 \pm 20$ & $14 \pm 3$ & $0.3 \pm 0.1$ & $7 \pm 1$ \\
\hline Without mucus & $59 \pm 19$ & $13 \pm 1$ & $0.1 \pm 0.1$ & $5 \pm 1$ \\
\hline
\end{tabular}

Gd was collected and measured by the ICP technique. The time point $72 \mathrm{~h}$ corresponds to $24 \mathrm{~h}$ exposure, followed by additional $48 \mathrm{~h}$ of culture. The results are presented as the percentage of the theoretical initial content 


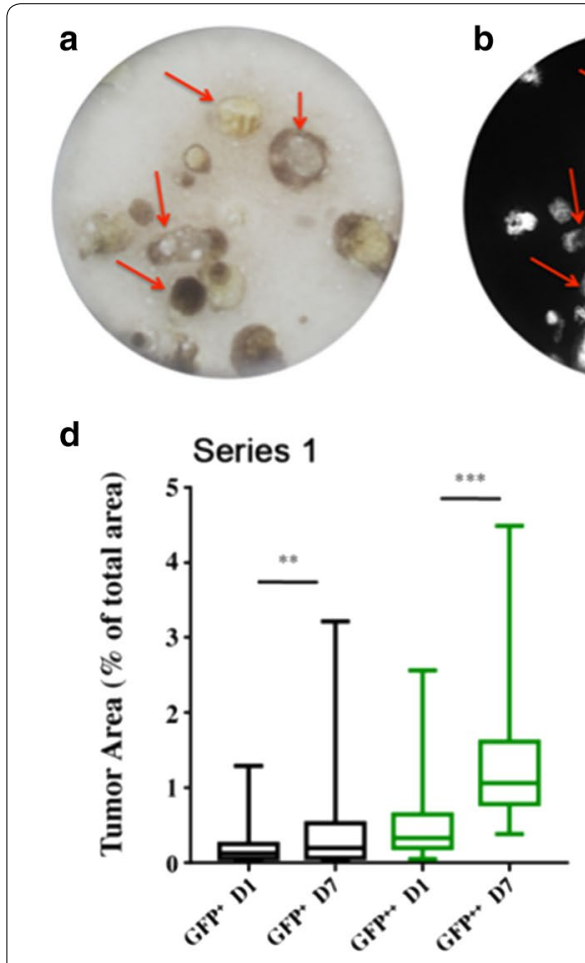

C

\begin{tabular}{|c|c|c|c|}
\hline Condition & $\begin{array}{c}\text { GFP-negative } \\
\text { Healthy cells }\end{array}$ & $\begin{array}{c}\mathrm{GFP}^{+} \text {Tumor } \\
\text { cells }\end{array}$ & $\begin{array}{c}\mathrm{GFP}^{++} \text {Tumor } \\
\text { cells }\end{array}$ \\
\hline 24 hours & $79 \pm 9 \%$ & $5 \pm 2 \%$ & $11 \pm 5 \%$ \\
\hline 72 hours & $74 \pm 12 \%$ & $6 \pm 4 \%$ & $13 \pm 8 \%$ \\
\hline
\end{tabular}

e

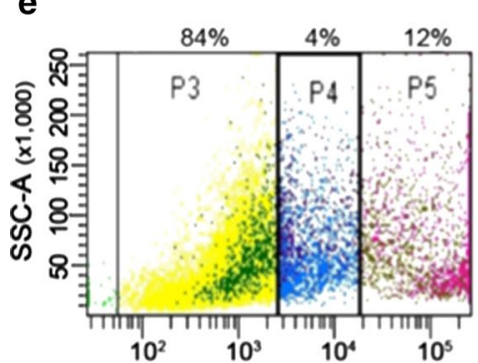

GFP-cell distribution

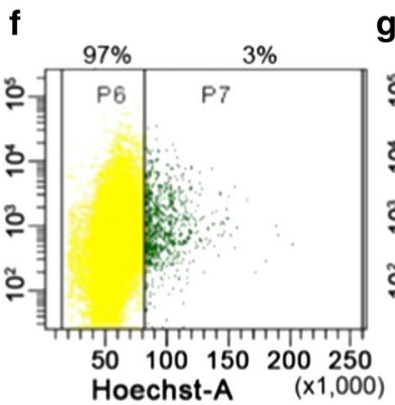

Healthy
Series 2

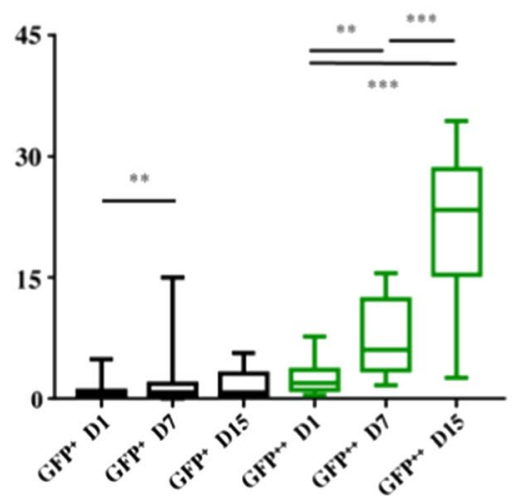

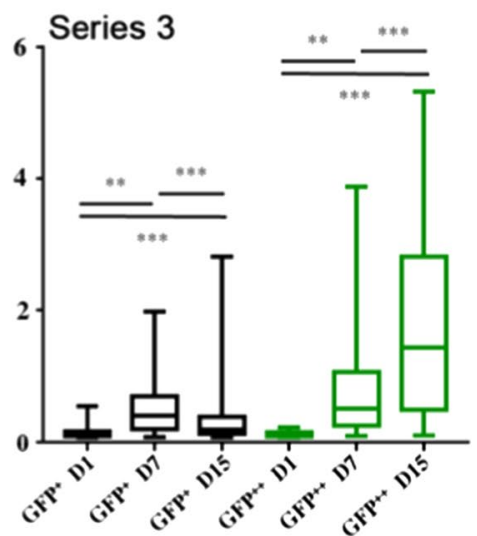

g

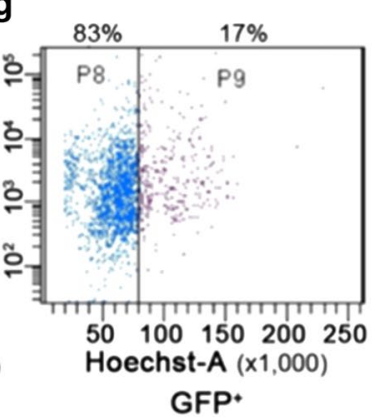

h

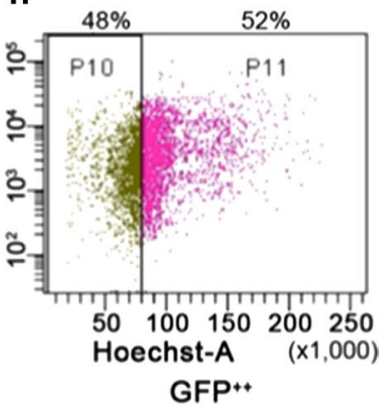

Fig. 3 Characterization of the A549-OncoCilAir ${ }^{\mathrm{TM}}$ model revealed different cell proliferation profiles. Representative pictures under white light (a) and GFP-fluorescence (b) of the cultures, on a same insert. Tumor cell evolution according to GFP expression by FCM analysis during AGuIX ${ }^{\circledR}$ NP's exposure (c), monitored by microscopy for 2 weeks (d), and an example of FCM cell cycle analysis (e-h). d Boxplots (line: median, box: 25-75\% percentiles, and whiskers: min-max) of the temporal evolution of relative tumor areas in 3 independent experiments. Bars indicate significant differences between groups (Tukey's test, ${ }^{*} p<0.05,{ }^{*} p<0.01,{ }^{* * *} p<0.001$ ). e Example of the tumor cell subpopulations as a function of GFP status $(n=6)$. The 3 subpopulations containing no $(P 3, \mathbf{f})$, low $(P 4, \mathbf{g})$, or high (P5, h) levels of GFP fluorescence were separated and analyzed for cell cycle proliferation using Hoechst staining $(n \geq 9)$. The quiescent (P6, P8 and P10) and proliferative (P7, P9, and P11) subpopulations are shown. The different percentages of cells are indicated above each panel for this representative example

$(47 \pm 7 \%)$ populations, which were more proliferative. This cell cycle analysis demonstrates that first, the majority of the healthy cells are quiescent, which is expected for epithelial differentiated cells, and second, the $\mathrm{GFP}^{+}$ and $\mathrm{GFP}^{++}$subpopulations are characterized by different proliferative capacities. Indeed, the $\mathrm{GFP}^{++}$tumor cells were approximately 2.5 times more proliferative than the $\mathrm{GFP}^{+}$cells, which may lead to differential AGuIX ${ }^{\circledR} \mathrm{NP}$ uptake according to proliferation rate.

\section{Distribution and quantification of AGuIX ${ }^{\circledR}$ NPs} in the OncoCilAir ${ }^{\mathrm{TM}}$ model

Similar to the experiments with MucilAir ${ }^{\mathrm{TM}}$, the distribution of $\mathrm{AGuIX}^{\circledR}$ NPs was investigated in OncoCilAir ${ }^{\mathrm{TM}}$ (3 independent experiments), with or without apical mucus. FCM analysis allowed the observation of the 3 subpopulations over time, as shown in Fig. 3. As previously observed for the MucilAir ${ }^{\mathrm{TM}}$ cultures, the presence of mucus did not have an impact on $\mathrm{AGuIX}^{\circledR} \mathrm{NP}$ 
internalization on OncocilAir ${ }^{\mathrm{TM}}$ cultures (Fig. 4a, merged data from apical exposure with and without mucus). In addition, AGuIX ${ }^{\circledR}$ NP's exposure did not affect the production of IL-8 or LDH release compared to control (data not shown). After a 24-hour exposure (Fig. 4b-e), AGuIX $^{\circledR}$ NPs were preferentially detected in the tumor subpopulation, with $\approx 80 \% \mathrm{AGuIX}^{\circledR}$-positive/GFP-positive cells compared to $\approx 40 \%$ in normal healthy surrounding epithelial cells, and were associated with a significantly higher mean fluorescence intensity (MFI). The GFP tumor subpopulations possessed similar AGuIX $^{\circledR}$ uptake profiles, both in terms of percentage of positive cells and uptake (MFI values). The relative uptake ratio was 2.4 to 2.8 -fold higher for tumor cells than for healthy cultures. The separate tumor proliferative capacity of $\mathrm{GFP}^{+}$versus $\mathrm{GFP}^{++}$cells did not clearly correlate with the uptake of NPs under our conditions.

After $72 \mathrm{~h}$ (Fig. $4 \mathrm{f}-\mathrm{i}$ ), the normal GFP-negative pool showed an equivalent percentage of AGuIX ${ }^{\circledR}$-positive and about $20 \%$ decrease in $\mathrm{AGuIX}^{\circledR}$-positive fluorescence intensity as compared to 24-h. Moreover, the NPs were released from a fraction of the tumor cells, as only $\approx 70 \%$ of the $\mathrm{GFP}^{+/++}$cells were $\mathrm{AGuIX}^{\circledR}$-positive, i.e., $10-15 \%$ less than at the 24-h time point. Such a reduction occurred concomitantly with a decrease in the MFI values about $30 \%$ both for $\mathrm{GFP}^{+}$and $\mathrm{GFP}{ }^{++}$populations

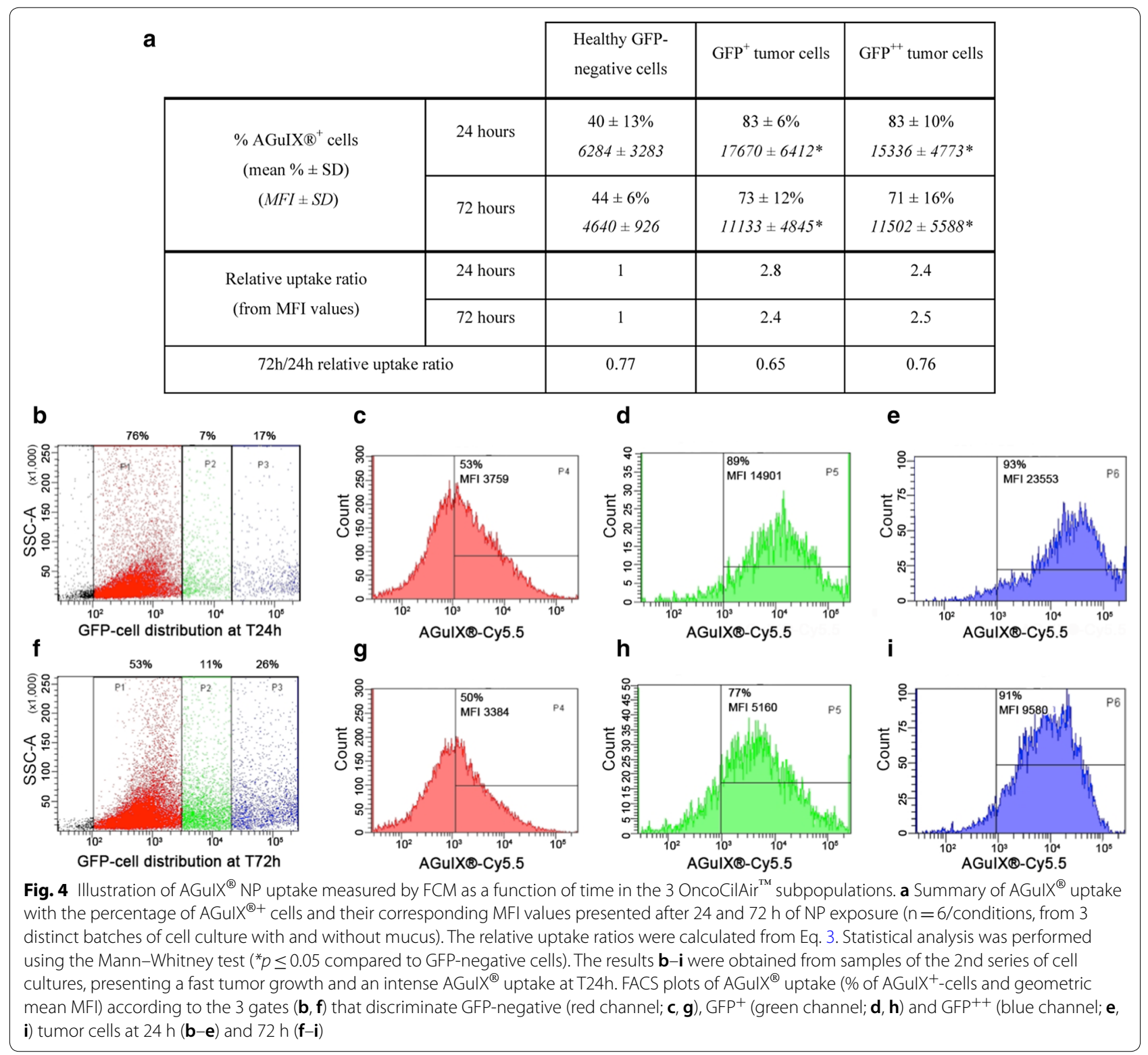


(See figure on next page.)

Fig. 5 Representative confocal images of OncoCilAir ${ }^{\mathrm{TM}}$ inserts $24 \mathrm{~h}$ after AGul $\mathrm{X}^{\circledR}-\mathrm{Cy} 5.5$ exposure. a AGuIX ${ }^{\circledR} \mathrm{NPs}$ were preferentially observed in tumor areas. The GFP+/++ tumor cells (green) and healthy tissues were labeled with ethidium bromide to show nuclei (blue). AGul ${ }^{\circledR}$-Cy5.5 NPs are represented in red. $\mathbf{b}$ Phalloidin staining (gray) revealed the organized healthy tissue compared to the chaotic tumor areas. $\mathbf{c}$ At higher magnification, the GFP/phalloidin colocalization confirmed AGuIX ${ }^{\circledR} N P$ internalization inside tumor cells: the NPs were clustered into cytoplasmic vesicles. All images are orthogonal views presenting the XY, XZ and $Y Z$ planes. $\mathbf{d}$ Without cell fixation and permeabilization, AGul $X^{\circledR}$-Cy5.5 NPs were also observed inside live tumor cells with clear evidence of NP internalization in cytoplasmic vesicles. The two first insets of $\mathbf{d}$ are 3D projection, while the two last are orthogonal views with different zooms. e A zoom-in at the interface between tumor and normal surrounding tissue evidenced the NP's behavior. The arrows indicated the presence of NP clusters in the normal tissue. Scale bar: $50 \mu m$

confirming a partial release of NPs during this 48 h-time period (Fig. 4a). However, according to the AGuIX ${ }^{\circledR}$ NP relative uptake ratios at 72 and $24 \mathrm{~h}$ for each population, the level of $\mathrm{AGuIX}^{\circledR}$ internalization into $\mathrm{GFP}^{+/++}$tumor areas remained 2.5 times higher than that in normal surrounding airway epithelium, as shown by the FCM quantification.

This efficient AGuIX ${ }^{\circledR}$ NP uptake by tumor cells was confirmed by confocal microscopy. As illustrated in Fig. 5, AGuIX ${ }^{\circledR}$ NPs were strongly internalized in tumor cells after $24 \mathrm{~h}$ of exposure, and the healthy surrounding environment remained almost unlabeled. The phalloidin labeling revealed the regular cell organization of the healthy airway environment, while the tumor areas were more chaotic and heterogeneous in terms of size and shape, as expected for tumor cell architecture. In Fig. 5a, one should note the NP accumulation in both the large compact and dense tumor area (right side) and the more diffuse tumor organization (left bottom). In addition, the higher magnification images showed NP internalization in small cytoplasmic vesicles (Fig. 5c, d, see also Additional file 1: Figure S4), as previously reported for tumor cell internalization in vivo, and in some peri-nuclear vesicles [4]. In a zoom-in at the interface between tumor and normal surrounding tissue, some internalized NPs were evidenced using increased contrast (Fig. 5e).

The ICP quantification of the NPs confirmed the strong passage of NPs through the OncoCilAir ${ }^{\mathrm{TM}}$ epithelium after apical exposure, as almost $\approx 20 \%$ of the particles were detected in the apical fraction at 24 -h, while $\approx 40 \%$ were present in the basal compartment (Additional file 1: Table S1). This passage from the apical to the basal compartment was almost threefold higher in the OncoCilAir $^{\mathrm{TM}}$ cultures with tumor cells than in the normal MucilAir $^{\mathrm{TM}}$ cultures. At $72 \mathrm{~h}$, the results were similar to those in the MucilAir ${ }^{\mathrm{TM}}$ cultures (Table 2), with $\approx 6 / 7 \%$ of the NPs released in the basal fraction and $\approx 1 \%$ in the apical fraction. The high NP passage into the basal compartment might be due to the presence of tumor cells, which affect the integrity of the airway epithelium. As indicated by the weak TEER values of the OncoCilAir ${ }^{\text {TM }}$ epithelium (see "Characterization of the OncoCilAir" ${ }^{\mathrm{TM}}$ airway model" section), the culture's integrity is modified by tumor areas, perhaps leading to efficient paracellular transport. This observation was correlated with cytometry analysis (Fig. 4a), where AGuIX ${ }^{\circledR}$ uptake was quantified on OncoCilAir ${ }^{\mathrm{TM}}$ cultures for about $40 \%$ of GFP-negative healthy cells against $15 \%$ in MucilAir ${ }^{\mathrm{TM}}$ cul- $^{-}$ tures (Fig. 1f).

\section{Radiosensitizing effect of AGuIX ${ }^{\circledR}$ NPs on tumor cells and the OncoCilAir ${ }^{\mathrm{TM}}$ model}

The radiosensitizing effect of AGuIX ${ }^{\circledR}$ NPs was evaluated on OncoCilAir ${ }^{\mathrm{TM}}$ cultures after a 24-hour apical contact followed by exposure to 4 Gy radiation. Tumor growth (Fig. 6a) is expressed as the percentage of the area covered by tumor GFP-cells in the cultures. As indicated in Fig. 6b, the combination of AGuIX ${ }^{\circledR}$ NPs and irradiation significantly reduced tumor growth compared to other conditions $(p<0.05 v s$. control or irradiation alone). The overall tumor growth showed a mean reduction of $18 \%$ compared to the control. Subtherapeutic radiation with a single 4 Gy dose reduced tumor growth in the absence of NPs, although this reduction did not reach significance, before tumor growth relapsed (after day-10). In addition, LDH release and IL-8 production were monitored at days 1, 2 and 3 post exposure (data not shown). The results indicated the absence of cytotoxicity, with $\mathrm{LDH}$ release $<5 \%$ and counterpart IL-8 production compared to controls $(4.000-4.500 \mathrm{pg} / \mathrm{mL})$ under all conditions. One should note that the basal level of IL-8 production in unexposed OncoCilAir ${ }^{\mathrm{TM}}$ cultures was significantly increased compared to MucilAir ${ }^{\mathrm{TM}}$ cultures $\left(p=0.002^{*}\right)$.

To better understand the biological impact of the AGuIX $^{\circledR}$ NPs, the experiment was repeated in vitro on GFP-A549 2D cultures alone after 4 Gy exposure. In 2D cell culture, the NPs in combination with irradiation significantly reduced cell viability compared to irradiation alone (Fig. 6c). Notably, the combination of NPs and 4 Gy exposure lead to similar cell growth as 6 Gy exposure alone in this 2D cell model (Additional file 1: Figure S5). In addition, DNA double-strand breaks were observed using $\gamma \mathrm{H} 2 \mathrm{AX}$ labeling (Fig. 6d-h). Exposure to 4 Gy radiation induced an increase in DNA double-strand breaks that doubled in the presence of NPs. In addition, the distribution of DNA double-strand breaks was determined 

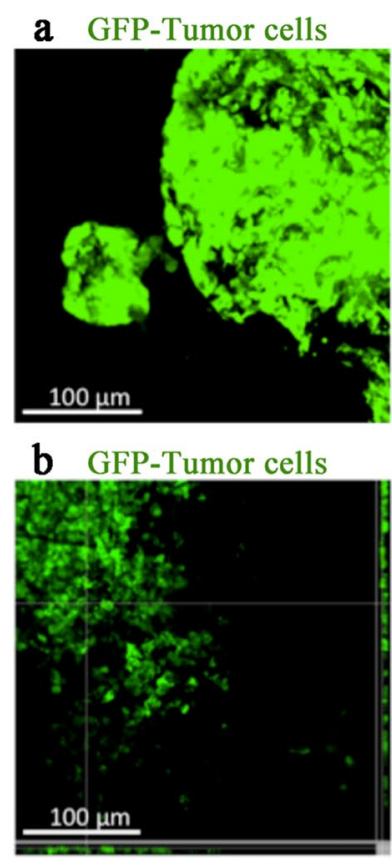

Phalloïdin/cell membrane
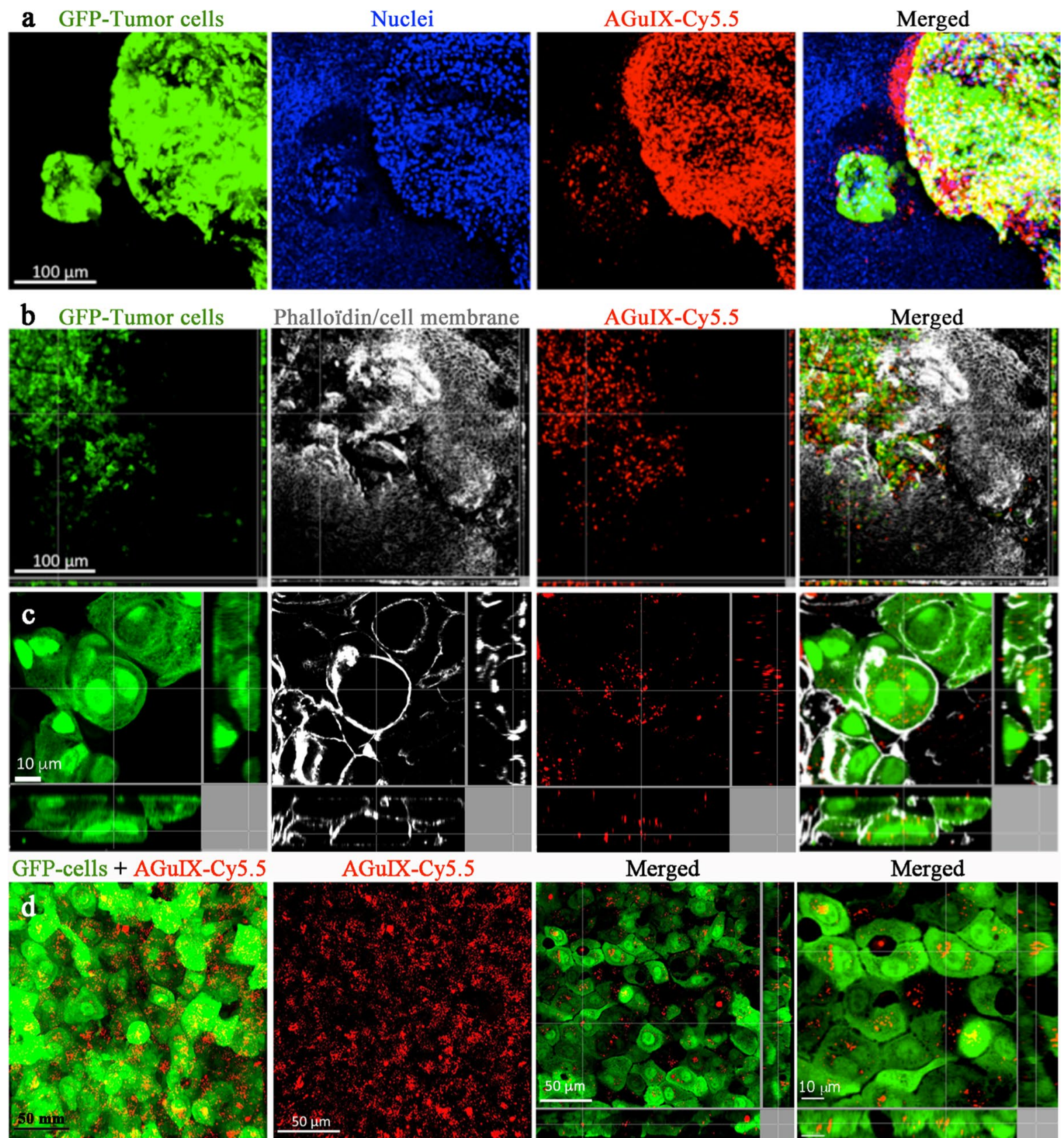

AGuLX-Cy5.5
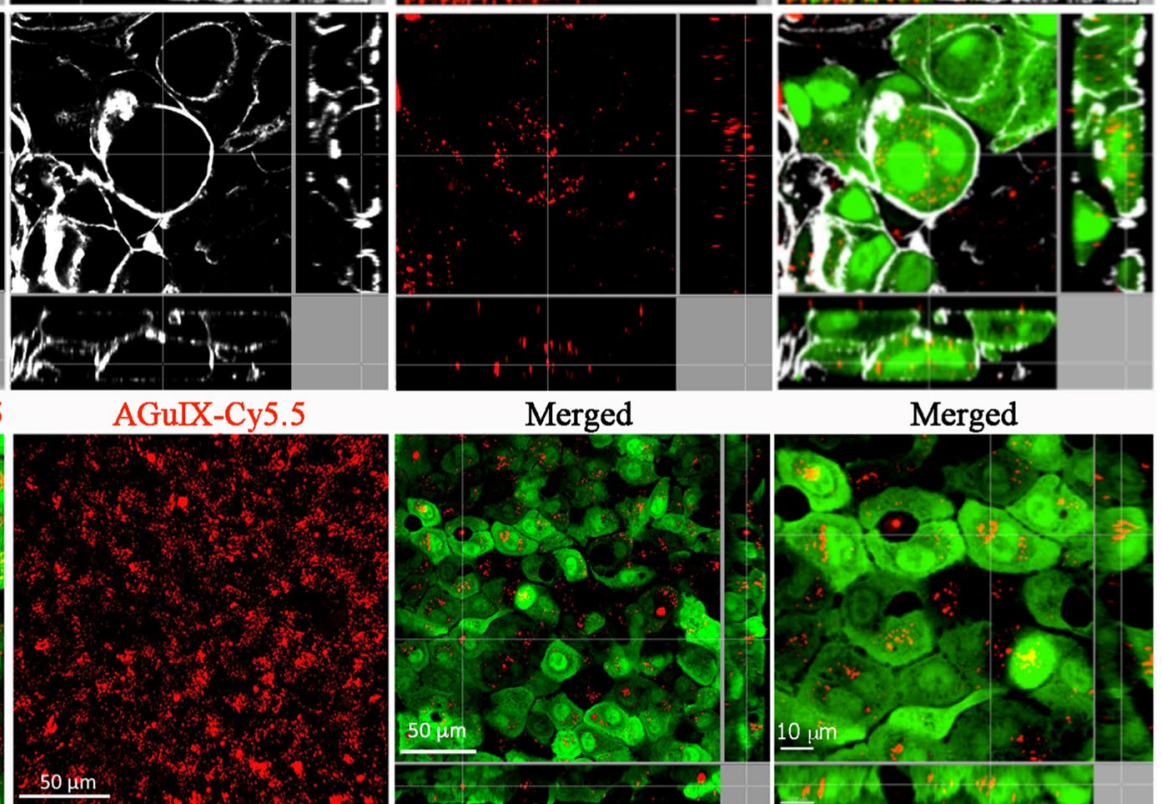

Merged

Merged

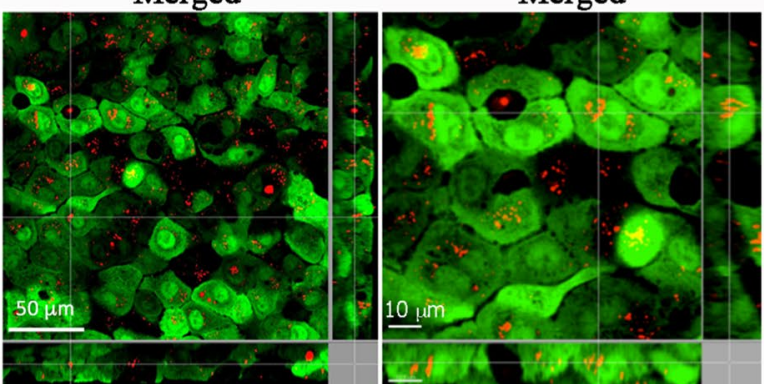

e

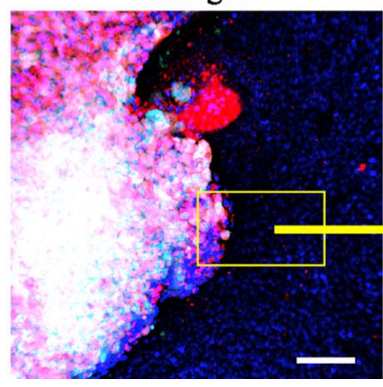

Zoom-in in the merged at the interface tumor/normal culture

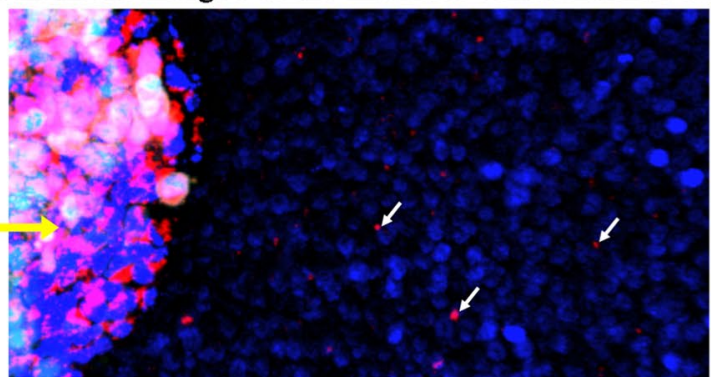




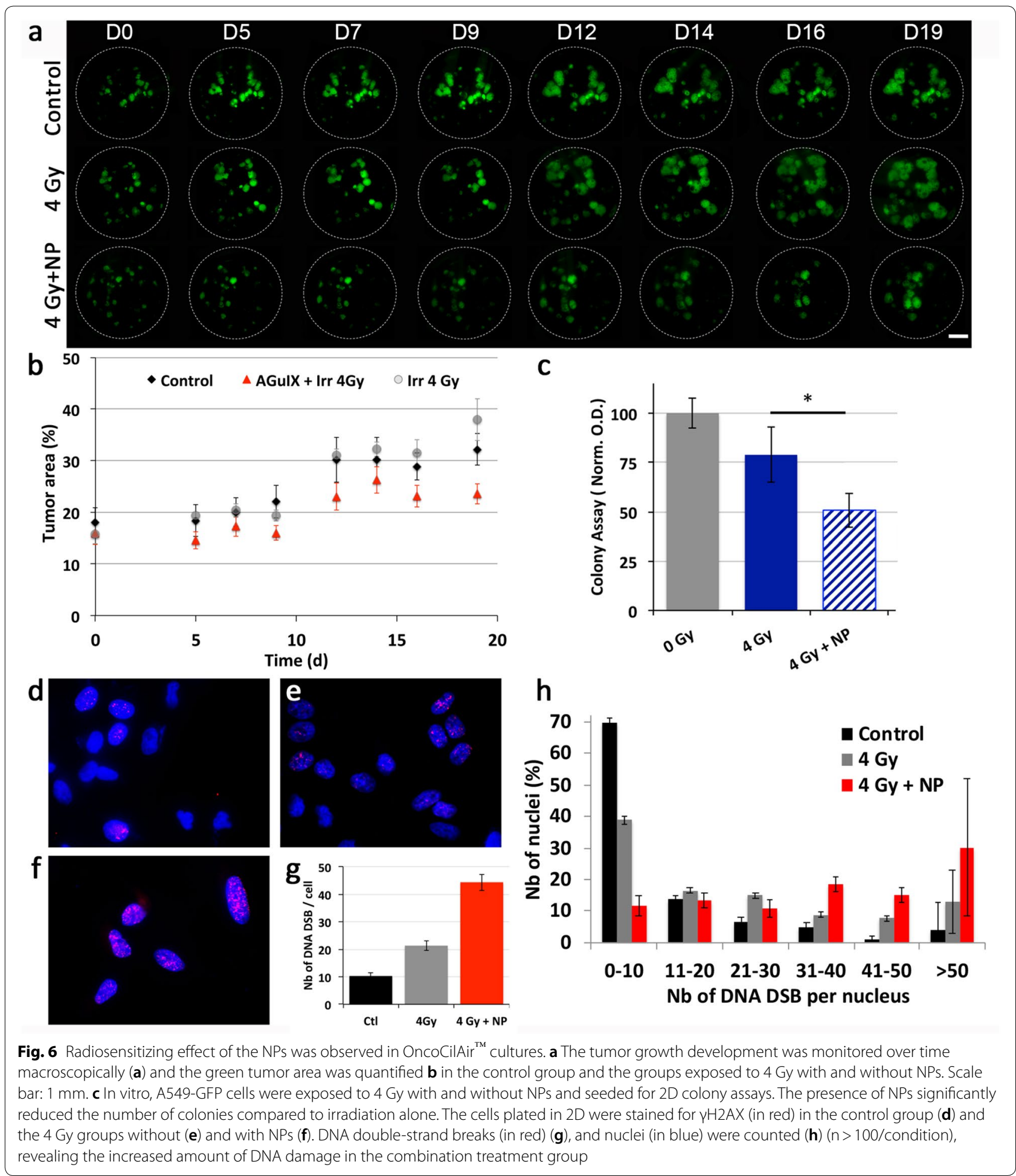

under different conditions: such breaks were more numerous in the combined NP and irradiation treatment group than in the irradiation alone group.

\section{Discussion}

From the perspective of bronchopulmonary cancer enhanced by radiation therapy, we investigated the safety, uptake, distribution, and radiosensitizing effect 
of ultrasmall gadolinium-based AGuIX ${ }^{\circledR}$ NPs on human healthy 3D-cultured (MucilAir ${ }^{\mathrm{TM}}$ ) and human tumorbearing epithelia (OncocilAir ${ }^{\mathrm{TM}}$ ). We used a multiple quantitative and qualitative approach to better compare and obtain information related to behavior.

As previously shown in the nasal MucilAir ${ }^{\mathrm{TM}}$ model [25], the bronchial pools of donors used to create MucilAir $^{\text {TM }}$ cultures mimic the morpho-functional characteristics of the human epi-airway with very good histological representation and mucus biological interface production. The intercellular cohesion of this epithelial model is principally ensured by the presence of tight junctions in the apical pole, which can be evaluated according to TEER and Papp measurements [25, 38]. Here, the different toxicity parameters studied in MucilAir ${ }^{\mathrm{TM}}$ cultures, including TEER monitoring, LDH release, IL-8 production, permeability (Papp), mucociliary clearance, and CBF did not show any acute toxicity, regardless of the presence of mucus, as previously observed for other preclinical models $[6,39,42]$. Interestingly, mucus did not affect the distribution of hybrid NPs. As previously reported, the penetration of small NPs inside respiratory mucus has been observed for particles up to $200 / 500 \mathrm{~nm}$, while larger NPs (>500 nm) mainly remain entrapped in the mucus [43-45]. NP penetration is related to NP size, and smaller NPs $(<60 \mathrm{~nm}$ size) were reported to pass through mucus pores. In addition, some particles, including those coated with PEG-moieties, might be mucoinert: these NPs do not interact with the mucus itself but are able to diffuse throughout mucus. In our conditions, the very small AGuIX ${ }^{\circledR}$ NPs $(<5 \mathrm{~nm})$ might possess such diffusing properties that facilitate their passage through mucus. Contrariwise, sub-micrometric $\mathrm{CeO}_{2} \mathrm{NPs}$ aggregated in presence of mucus, reducing their transport across the respiratory mucosa [29].

The AGuIX ${ }^{\circledR}$ NPs accumulated weakly in the healthy airway epithelium MucilAir ${ }^{\mathrm{TM}}$ and diffused into the basal compartment. NP diffusion might occur through transcellular way, as indicated by the microscopy observations, barrier integrity with high TEER values and according to $\mathrm{AGuIX}^{\circledR}$ permeation analysis (Papp measurements). Moreover, these observations also were in agreement with a toxico-kinetic MucilAir ${ }^{\mathrm{TM}}$ study, demonstrating that organic chemicals have a higher permeability when compared to inorganic compounds [38]. In addition, the very small NP size $(<5 \mathrm{~nm})$ reinforces this observation. Furthermore, as demonstrated by the combined FCM and microscope analysis, ciliated cells are mainly involved in $\mathrm{AGuIX}^{\circledR} \mathrm{NP}$ uptake with a preferential occurrence at the apical pole of cilia cells, between motile cilia cells, with accumulation at the cilia anchoring area. This observation was in agreement with the principal function of surface exchange of this cell type. Such data are also in agreement with previous observations in vivo after the orotracheal administration of nanoparticles in mice bearing lung tumors. In this previous work, the nanoparticles strongly accumulated inside the tumors, while they were washed out of healthy tissues [19].

The distribution studies performed in OncoCilAir ${ }^{\mathrm{TM}}$ cultures confirmed a narrow NP uptake by healthy cells, uptake that was slightly higher as compared to the one of MucilAir ${ }^{\mathrm{TM}}$ cultures. Indeed, in OncoCilAir $^{\mathrm{TM}}$ cultures, the A549 tumor microenvironment affects integrity of epithelial barrier, as indicated by the low TEER values, which might contribute to increase healthy cell uptake by promoting paracellular transport due to the reduction in intercellular tight junction bridges. Nevertheless, the NPs robustly accumulated in small cytoplasmic vesicles in tumor cells, as previously observed for subcutaneous tumors, for a prolonged time [4]: AGuIX ${ }^{\circledR}$ NP fluorescence was still evident by FCM $72 \mathrm{~h}$ after NP exposure. The extended and high tumor cell uptake is an advantage for any clinical application in lung tumor enhanced radiotherapy: considering the unique administration of NPs in the airways, radiosensitizing NPs may stay inside the tumor region for several days to improve efficacy over several daily radiation exposures $[6,46]$.

The radiosensitizing effect of the gadolinium-based AGuIX $^{\circledR}$ NPs has been evidenced in various pathologies including lung tumors $[4,6,46]$. In this article, the radiosensitizing effect was observed for a single radiation exposure $24 \mathrm{~h}$ after the local administration of NPs, on OncoCilAir $^{\mathrm{TM}}$ cultures. The combination of AGuIX ${ }^{\circledR}$ NPs and 4 Gy irradiation has reduced significantly the surface tumor area compared to the irradiation alone. Interestingly, the 4 Gy radiosensitizing effect observed in the presence of NPs was similar to that measured after a 6 Gy exposure, on 2D-A549 cells (see Additional file 1: Figure S5). This result might indicate that a reduction in the exposure dose might be possible for a similar antitumor treatment for radiosensitive tumors or that radioresistant tumors might be more efficiently treated. In addition, the observed radiosensitizing effect might be increased in vivo, where the tumor lesions are vascularized. Indeed, in vivo, the $\mathrm{AGuIX}^{\circledR}$ NPs might cross the epithelium and enter the vascular network, leading to further tumor accumulation though the EPR effect $[4,12]$. The AGuIX ${ }^{\circledR}$ NPs are not targeted NPs but passively accumulate inside tumor areas, as previously described in vitro and in vivo $[4,12,13,47,48]$. In this static in vitro culture system, the non-functionalized AGuIX ${ }^{\circledR}$ NPs were preferentially internalized in tumor cells relatively to healthy cells. Besides EPR effect, the cellular mechanism responsible of the differential in vitro uptake should be further explored; we observed that AGuIX ${ }^{\circledR}$ internalization was not related to tumor cell proliferation (Fig. 3), but might 
be due to active membrane transport and tumor cell permeability, as previously observed by macropinocytosis investigations [49]. This may indicate efficient AGuIX ${ }^{\circledR}$ NP accumulation inside both highly and poorly proliferative tumors, similar to the cancer heterogeneity observed in the clinic.

To the best of our knowledge, this in vitro study reports for the first time the toxicity and distribution of NPs dedicated to theranostic applications, i.e., AGuIX ${ }^{\circledR}$ NPs in both a healthy and an onco-airway 3D epithelium model. We demonstrate that healthy MucilAir ${ }^{\mathrm{TM}}$ cultures constitute a robust model for fundamental and applicable investigations of NP behavior due to homogeneity among different cultures, achieved by pooling cells from human donors [49]. Additionally, the impact of mucus, which constitutes an overriding biological interface for NP-cell interactions, might be studied in such an in vitro model $[24,25]$. Under our conditions, the mucus had no impact on the uptake or the toxicity of the AGuIX ${ }^{\circledR}$ NPs.

OncoCilAir $^{\mathrm{TM}}$ cultures are a powerful in vitro model of 3D tissue for the assessment of anticancer therapy, as these cultures integrates A549 tumor cells representative of lung adenocarcinoma inside a healthy and functional airway tissue. While previous studies based on molecular compounds have already been performed on this type of model, this is the first study related to nanomedicine for oncology [30].

\section{Conclusion}

For the first time, solid theranostic AGuIX ${ }^{\circledR}$ NPs have been investigated in in vitro airway epithelium models with and without tumor areas. These valuable tools for bronchopulmonary investigations and anticancer therapies may bridge the gap between 2D cultures and human applications, allowing a reduction in animal testing while mimicking human behaviors. In these models, AGuIX ${ }^{\circledR}$ NPs did not present evidence of toxicity, even at high local concentrations. The NPs preferentially accumulated in the tumor areas, where they were able to exert their radiosensitizing effect upon X-ray exposure. In the context of personalized medicine and enhanced radiation therapies for lung cancer, the administration of AGuIX ${ }^{\circledR}$ NPs by aerosolization appears to be a promising and relevant approach.

\section{Supplementary information}

Supplementary information accompanies this paper at https://doi. org/10.1186/s12951-020-00683-6.

Additional file 1: Figure S1. Main AGuIX characteristics. Figure S2. MucilAir $^{\mathrm{TM}}$ and OncoCilAir ${ }^{\mathrm{TM}}$ histological and phenotypic characteristics. Figure S3. Complementary representative confocal images of OncoCilAir ${ }^{\mathrm{TM}}$ inserts $24 \mathrm{~h}$ after AGuIX ${ }^{\circledR}-$ Cy5.5 exposure. Figure S4.
Radiosensitizing effect of the nanoparticles in combination with radiation exposure. Table S1. AGuIX ${ }^{\circledR}$ NPs quantification by ICP-MS on OncoCilAir ${ }^{\text {TM }}$ tissue.

\section{Acknowledgements}

The authors acknowledge the support of the French Agence Nationale de la Recherche for the ANR project Multimage (ANR-12-RPIB-0010). The authors also acknowledge NH TherAguix for furnishing AGuI ${ }^{\circledR}$ preclinical nanoparticles, and Sophie Hodin and Malika Dakir for their technical contributions.

\section{Authors' contributions}

$D B$ and $L S$ designed and conceived the experiments. $D B, L S, O S, Z H, F R, A G$, $\mathrm{VB}, \mathrm{CM}$, and SC performed the experiments and/or analyzed the data. FL and OT produced the particles. SC provided the airway epithelia cultures MuciIAir $^{\mathrm{TM}}$ and C.M. produced the tumor lung cultures OncoCilAir ${ }^{\mathrm{TM}}$. FL, OT, JLC, and $M C$ provided technical support and conceptual advices. LS and DB wrote the manuscript. All the authors contributed to the final manuscript. All authors read and approved the final manuscript.

\section{Competing interests}

FL. and O.T. have to disclose the patent WO2011/135101. O.T. has to disclose the patent WO2009/053644. These patents protect the AGuIX ${ }^{\circledR}$ NPs described in this publication. F.L. and O.T. are employees from NH TherAguix that is developing the AGuIX ${ }^{\circledR} N$ NS. FL, OT, LS and J-L. C. possess shares in NH TherAguix company. C.M. and S.C. are employees from Epithelix/OncoTheis that is developing and providing the epithelium cultures.

\section{Author details}

${ }^{1}$ Institute for Advanced Biosciences, INSERM U1209, CNRS, UMR 5309, Université Grenoble Alpes, 38000 Grenoble, France. ${ }^{2}$ INSERM U1059, Laboratoire SAINBIOSE, équipe DVH/PIB, Faculté de Médecine, Université Jean Monnet, Saint-Etienne, France. ${ }^{3}$ Université de Lyon, Saint-Etienne, France. ${ }^{4}$ BiiGC EA2521, Saint-Etienne, France. ${ }^{5}$ Institut Lumière Matière, CNRS UMR5306, Université Lyon 1, 69100 Villeurbanne, France. ${ }^{6}$ SAINBIOSE, Inserm U1059, LBTO Team, Saint-Etienne, France. ${ }^{7}$ CHU Saint Etienne, Hôpital Nord, UF6725 Cytologie et Histologie Rénale, St-Etienne, France. ${ }^{8} \mathrm{NH}$ Theraguix, 38240 Meylan, France. ${ }^{9}$ Institut Universitaire de France (IUF), Paris, France. ${ }^{10}$ Epithelix SARL, Geneva, Switzerland. ${ }^{11}$ OncoTheis SARL, Geneva, Switzerland.

Received: 11 May 2020 Accepted: 26 August 2020

Published online: 10 September 2020

\section{References}

1. Falkson CB, Vella ET, Yu E, El-Mallah M, Mackenzie R, Ellis PM, et al. Guideline for radiotherapy with curative intent in patients with early-stage medically inoperable non-small-cell lung cancer. Curr Oncol. 2017;24(1):e44-9.

2. Shah JL, Loo BW Jr. Stereotactic ablative radiotherapy for early-stage lung cancer. Semin Radiat Oncol. 2017;27(3):218-28.

3. Maciejczyk A, Skrzypczynska I, Janiszewska M. Lung cancer. Radiotherapy in lung cancer: Actual methods and future trends. Rep Pract Oncol Radiother. 2014;19(6):353-60.

4. Kotb S, Detappe A, Lux F, Appaix F, Barbier EL, Tran VL, et al. Gadoliniumbased nanoparticles and radiation therapy for multiple brain melanoma metastases: proof of concept before phase I trial. Theranostics. 2016;6(3):418-27.

5. Le Duc G, Miladi I, Alric C, Mowat P, Brauer-Krisch E, Bouchet A, et al. Toward an image-guided microbeam radiation therapy using gadolinium-based nanoparticles. ACS Nano. 2011;5(12):9566-74.

6. Verry C, Dufort S, Barbier EL, Montigon O, Peoc'h M, Chartier P, et al. MRI-guided clinical 6-MV radiosensitization of glioma using a unique gadolinium-based nanoparticles injection. Nanomedicine (Lond). 2016;11(18):2405-17.

7. LuX F, Tran VL, Thomas E, Dufort S, Rossetti F, Martini M, et al. AGuIX((R)) from bench to bedside-Transfer of an ultrasmall theranostic gadoliniumbased nanoparticle to clinical medicine. Br J Radiol. 2018;2018:365.

8. Maggiorella L, Barouch G, Devaux C, Pottier A, Deutsch E, Bourhis J, et al. Nanoscale radiotherapy with hafnium oxide nanoparticles. Future Oncol. 2012;8(9):1167-81. 
9. Berbeco Rl, Detappe A, Tsiamas P, Parsons D, Yewondwossen M, Robar J. Low Z target switching to increase tumor endothelial cell dose enhancement during gold nanoparticle-aided radiation therapy. Med Phys. 2016;43(1):436.

10. Hainfeld JF, Smilowitz HM, O'Connor MJ, Dilmanian FA, Slatkin DN. Gold nanoparticle imaging and radiotherapy of brain tumors in mice. Nanomedicine (Lond). 2013;8(10):1601-9.

11. Bianchi A, Dufort S, Fortin PY, Lux F, Raffard G, Tassali N, et al. In vivo MRI for effective non-invasive detection and follow-up of an orthotopic mouse model of lung cancer. NMR Biomed. 2014;27(8):971-9.

12. Dufort S, Bianchi A, Henry M, Lux F, Le Duc G, Josserand V, et al. Nebulized gadolinium-based nanoparticles: a theranostic approach for lung tumor imaging and radiosensitization. Small. 2015;11(2):215-21.

13. Sancey L, Lux F, Kotb S, Roux S, Dufort S, Bianchi A, et al. The use of theranostic gadolinium-based nanoprobes to improve radiotherapy efficacy. Br J Radiol. 2014;87(1041):20140134.

14. Ishiguro S, Cai S, Uppalapati D, Turner K, Zhang T, Forrest WC, et al. Intratracheal administration of hyaluronan-cisplatin conjugate nanoparticles significantly attenuates lung cancer growth in mice. Pharm Res. 2016;33(10):2517-29.

15. Roa WH, Azarmi S, Al-Hallak MH, Finlay WH, Magliocco AM, Lobenberg R. Inhalable nanoparticles, a non-invasive approach to treat lung cancer in a mouse model. J Control Release. 2011;150(1):49-55.

16. Karageorgis A, Dufort S, Sancey L, Henry M, Hirsjarvi S, Passirani C, et al. An MRI-based classification scheme to predict passive access of 5 to 50-nm large nanoparticles to tumors. Sci Rep. 2016;6:21417.

17. Aillon KL, El-Gendy N, Dennis C, Norenberg JP, McDonald J, Berkland C. lodinated NanoClusters as an inhaled computed tomography contrast agent for lung visualization. Mol Pharm. 2010;7(4):1274-82.

18. Bianchi A, Dufort S, Lux F, Courtois A, Tillement O, Coll JL, et al. Quantitative biodistribution and pharmacokinetics of multimodal gadoliniumbased nanoparticles for lungs using ultrashort TE MRI. MAGMA. 2014:27(4):303-16.

19. Bianchi A, Dufort S, Lux F, Fortin PY, Tassali N, Tillement O, et al. Targeting and in vivo imaging of non-small-cell lung cancer using nebulized multimodal contrast agents. Proc Natl Acad Sci USA. 2014;111(25):9247-52.

20. Verry C, Sancey L, Dufort S, Le Duc G, Mendoza C, Lux F, et al. Treatment of multiple brain metastases using gadolinium nanoparticles and radiotherapy: NANO-RAD, a phase I study protocol. BMJ Open. 2019;9(2):e023591.

21. Lux F, Mignot A, Mowat P, Louis C, Dufort S, Bernhard C, et al. Ultrasmall rigid particles as multimodal probes for medical applications. Angew Chem Int Ed Engl. 2011;50(51):12299-303.

22. Cremillieux Y, Montigaud Y, Bal C, Pinaud N, Pham V, Perinel S, et al. Three-dimensional quantitative MRI of aerosolized gadolinium-based nanoparticles and contrast agents in isolated ventilated porcine lungs. Magn Reson Med. 2020;83(5):1774-82.

23. Mas C, Boda B, Caul Futy M, Huang S, Wisniewski L, Constant S. Establishment of a tumour-stroma airway model (OncoCilAir) to accelerate the development of human therapies against lung cancer. Altern Lab Anim. 2016;44(5):479-85.

24. Huang S, Wiszniewski L, Constant S, Roggen E. Potential of in vitro reconstituted 3D human airway epithelia (MucilAir) to assess respiratory sensitizers. Toxicol In Vitro. 2013;27(3):1151-6.

25. Mercier C, He Z, Constant S, Perek N, Boudard D, Delavenne X. Pharmacological characterization of the 3D MucilAir ${ }^{\mathrm{TM}}$ nasal model. Eur J Pharm Biopharm. 2019;139:186-96.

26. Hoshino M, Morita S, Iwashita H, Sagiya Y, Nagi T, Nakanishi A, et al. Increased expression of the human $\mathrm{Ca}^{2+}$-activated $\mathrm{Cl}$ - channel 1 (CaCC1) gene in the asthmatic airway. Am J Respir Crit Care Med. 2002;165(8):1132-6.

27. Blatt EN, Yan XH, Wuerffel MK, Hamilos DL, Brody SL. Forkhead transcription factor HFH-4 expression is temporally related to ciliogenesis. Am J Respir Cell Mol Biol. 1999;21(2):168-76.

28. Jain R, Pan J, Driscoll JA, Wisner JW, Huang T, Gunsten SP, et al. Temporal relationship between primary and motile ciliogenesis in airway epithelial cells. Am J Respir Cell Mol Biol. 2010;43(6):731-9.

29. Frieke Kuper C, Grollers-Mulderij M, Maarschalkerweerd T, Meulendijks NM, Reus A, van Acker F, et al. Toxicity assessment of aggregated/agglomerated cerium oxide nanoparticles in an in vitro 3D airway model: the influence of mucociliary clearance. Toxicol In Vitro. 2015;29(2):389-97.
30. Meldrum K, Robertson SB, Romer I, Marczylo T, Dean LSN, Rogers A, et al. Cerium dioxide nanoparticles exacerbate house dust mite induced type ॥ airway inflammation. Part Fibre Toxicol. 2018;15(1):24.

31. Mas C, Boda B, CaulFuty M, Huang S, Wiszniewski L, Constant S. Antitumour efficacy of the selumetinib and trametinib MEK inhibitors in a combined human airway-tumour-stroma lung cancer model. J Biotechnol. 2015;205:111-9.

32. Chortarea S, Fytianos K, Rodriguez-Lorenzo L, Petri-Fink A, RothenRutishauser B. Distribution of polymer-coated gold nanoparticles in a $3 \mathrm{D}$ lung model and indication of apoptosis after repeated exposure. Nanomedicine (Lond). 2018;13(10):1169-85.

33. Drasler B, Vanhecke D, Rodriguez-Lorenzo L, Petri-Fink A, RothenRutishauser B. Quantifying nanoparticle cellular uptake: which method is best? Nanomedicine (Lond). 2017;12(10):1095-9.

34. Milosevic AM, Rodriguez-Lorenzo L, Balog S, Monnier CA, Petri-Fink A, Rothen-Rutishauser B. Assessing the stability of fluorescently encoded nanoparticles in lysosomes by using complementary methods. Angew Chem Int Ed Engl. 2017:56(43):13382-6.

35. Mignot A, Truillet C, Lux F, Sancey L, Louis C, Denat F, et al. A top-down synthesis route to ultrasmall multifunctional Gd-based silica nanoparticles for theranostic applications. Chemistry. 2013;19(19):6122-36.

36. Morlieras J, Dufort S, Sancey L, Truillet C, Mignot A, Rossetti F, et al. Functionalization of small rigid platforms with cyclic RGD peptides for targeting tumors overexpressing alphavbeta3-integrins. Bioconjug Chem. 2013:24(9):1584-97.

37. Huang S, Wiszniewski L, Constant $\mathrm{S}$. The use of in vitro $3 \mathrm{~d}$ cell models in drug development for respiratory diseases. In: Kapetanović I, editor. Drug Discovery and development—-present and future; 2011. p. 169-90.

38. Hoffmann W, Gradinaru J, Farcal L, Caul-Futy M, Huang S, Wiszniewski L, et al. Establishment of a human 3D tissue-based assay for upper respiratory tract absorption. Appl Vitro Toxicol. 2018:4:2.

39. Sancey L, Kotb S, Truillet C, Appaix F, Marais A, Thomas E, et al. Long-term in vivo clearance of gadolinium-based AGuIX nanoparticles and their biocompatibility after systemic injection. ACS Nano. 2015;9(3):2477-88.

40. Figarol A, Pourchez J, Boudard D, Forest V, Akono C, Tulliani JM, et al. In vitro toxicity of carbon nanotubes, nano-graphite and carbon black, similar impacts of acid functionalization. Toxicol In Vitro. 2015;30(1 Pt B):476-85

41. Kurtz-Chalot A, Villiers C, Pourchez J, Boudard D, Martini M, Marche PN, et al. Impact of silica nanoparticle surface chemistry on protein corona formation and consequential interactions with biological cells. Mater Sci Eng C Mater Biol Appl. 2017;75:16-24.

42. Dufort S, Sancey L, Coll JL. Physico-chemical parameters that govern nanoparticles fate also dictate rules for their molecular evolution. Adv Drug Deliv Rev. 2012;64(2):179-89.

43. Schneider CS, Xu Q, Boylan NJ, Chisholm J, Tang BC, Schuster BS, et al. Nanoparticles that do not adhere to mucus provide uniform and long-lasting drug delivery to airways following inhalation. Sci Adv. 2017;3(4):e1601556.

44. Schuster BS, Suk JS, Woodworth GF, Hanes J. Nanoparticle diffusion in respiratory mucus from humans without lung disease. Biomaterials. 2013;34(13):3439-46.

45. Lai SK, Wang YY, Hanes J. Mucus-penetrating nanoparticles for drug and gene delivery to mucosal tissues. Adv Drug Deliv Rev. 2009;61(2):158-71.

46. Dufort S, Le Duc G, Salome M, Bentivegna V, Sancey L, Brauer-Krisch E, et al. The high radiosensitizing efficiency of a trace of gadolinium-based nanoparticles in tumors. Sci Rep. 2016;6:29678.

47. Detappe A, Kunjachan S, Rottmann J, Robar J, Tsiamas P, Korideck H, et al. AGulX nanoparticles as a promising platform for image-guided radiation therapy. Cancer Nanotechnol. 2015;6(1):4.

48. Rima W, Sancey L, Aloy MT, Armandy E, Alcantara GB, Epicier T, et al. Internalization pathways into cancer cells of gadolinium-based radiosensitizing nanoparticles. Biomaterials. 2013;34(1):181-95.

49. Balogh Sivars K, Sivars U, Hornberg E, Zhang H, Branden L, Bonfante R, et al. A 3D human airway model enables prediction of respiratory toxicity of inhaled drugs in vitro. Toxicol Sci. 2018;162(1):301-8.

\section{Publisher's Note}

Springer Nature remains neutral with regard to jurisdictional claims in published maps and institutional affiliations. 